\section{(6) OPEN ACCESS}

\title{
Safety and efficacy of dabigatran versus warfarin in patients undergoing catheter ablation of atrial fibrillation: a systematic review and meta-analysis
}

\author{
Rui Providência, ${ }^{1,2}$ Jean-Paul Albenque, ${ }^{1}$ Stephane Combes, ${ }^{1}$ Abdeslam Bouzeman, ${ }^{1}$ \\ Benjamin Casteigt, ${ }^{1}$ Nicolas Combes, ${ }^{1}$ Kumar Narayanan, ${ }^{3}$ Eloi Marijon, ${ }^{3,4}$ \\ Serge Boveda ${ }^{1}$
}

'Département de Rythmologie, Clinique Pasteur, Toulouse, France

${ }^{2}$ Faculty of Medicine, University of Coimbra, Coimbra, Portugal ${ }^{3}$ Heart Institute, Cedars Sinai Medical Center, Los Angeles, California, USA

${ }^{4}$ Paris Cardiovascular Research Center, Paris, France

\section{Correspondence to} Dr Rui Providência, Département de Rythmologie, Clinique Pasteur, 45 avenue de Lombez, BP 27617, Toulouse, 31076 Cedex 3, France; rui_providencia@yahoo.com

Received 30 May 2013 Revised 1 July 2013 Accepted 2 July 2013 Published Online First 22 July 2013

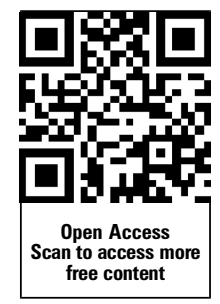

To cite: Providência $R$ Albenque J-P, Combes $S$, et al. Heart 2014:100: 324-335.

\section{ABSTRACT}

Background Dabigatran etexilate, a new thrombin inhibitor, has been shown to be comparable to warfarin in patients with atrial fibrillation (AF). However, there is a limited body of evidence on the efficacy and safety of using dabigatran among patients undergoing $\mathrm{AF}$ catheter ablation.

Objective A random effects meta-analysis was performed of controlled trials comparing dabigatran and warfarin in paroxysmal/persistent AF patients undergoing catheter ablation.

Methods Data sources included Medline, Embase, and Cochrane (from inception to April 2013). Three independent reviewers selected studies comparing warfarin to dabigatran. Descriptive and quantitative information was extracted from each selected study, regarding periprocedural all cause mortality, thromboembolic events and major bleeding, as well as modalities of periprocedural anticoagulation bridging. Results After a detailed screening of 228 search results, 14 studies were identified enrolling a total of 4782 patients (1823 treated with dabigatran and 2959 with warfarin). No deaths were reported. No significant differences were found between patients treated with dabigatran and warfarin as regards thromboembolic events $(0.55 \%$ dabigatran vs $0.17 \%$ warfarin; risk ratios $(R R)=1.78,95 \% \mathrm{Cl} 0.66$ to $4.80 ; p=0.26)$ and major bleeding $(1.48 \%$ dabigatran vs $1.35 \%$ warfarin; $\mathrm{RR}=1.07,95 \% \mathrm{Cl} 0.51$ to $2.26 ; p=0.86)$. No difference was found between the $110 \mathrm{mg}$ twice daily and $150 \mathrm{mg}$ twice daily dabigatran dosages concerning major bleeding ( $0 \%$ vs $1.62 \%$, respectively; $R R=0.19,95 \% \mathrm{Cl}$ 0.01 to $3.18 ; p=0.25$ ) and thromboembolism ( $0 \%$ vs $0.40 \%$, respectively; $R R=0.72,95 \% \mathrm{Cl} 0.04$ to 12.98 ; $\mathrm{p}=0.82$ ).

Conclusions In the specific setting of AF catheter ablation, this first pooled analysis suggests that patients treated with dabigatran have a similar incidence of thromboembolic events and major bleeding compared to warfarin, with low event rates overall.

\section{INTRODUCTION}

Atrial fibrillation (AF) is the most prevalent sustained arrhythmia and its prevalence is likely to rise steeply until $2050 .{ }^{1}$ Stroke and systemic embolism are among the most feared complications of $\mathrm{AF}$ and can be effectively tackled by anticoagulation. ${ }^{2}$

Catheter ablation is currently recommended (class IIa, level of evidence $\mathrm{C}$ ) as an interventional alternative for the treatment of patients with $\mathrm{AF}$ having symptomatic recurrences despite antiarrhythmic therapy. ${ }^{3}$ Over the last 13 years this has become a very commonly performed procedure for the treatment of symptomatic AF patients. ${ }^{4}$

Rigorous anticoagulation in the setting of $\mathrm{AF}$ catheter ablation has been demonstrated to be of prime importance. The recent large phase III trials involving the use of novel oral anticoagulants ${ }^{5-7}$ confirmed the non-inferiority, and even superiority in some cases, of dabigatran, rivaroxaban, and apixaban compared to warfarin in $\mathrm{AF}$ patients. However, since a planned AF catheter ablation procedure was listed as an exclusion criteria in those trials, the efficacy and safety results cannot be extended to that setting. The 2012 Expert Consensus Statement on the management of patients with AF, by the Heart Rhythm Society, European Heart Rhythm Association, and European Cardiac Arrhythmia Society, ${ }^{8}$ highlights the limited clinical experience with the new anticoagulants in AF catheter ablation. Thus, even though dabigatran is being widely used and has been in vogue longer than the other new agents, strong evidence towards its utilisation in patients undergoing $\mathrm{AF}$ ablation is lacking. Further, the presence of controversial results ${ }^{9}$ in controlled trials with warfarin has led cardiologists to question its safety and efficacy.

We therefore aimed to systematically evaluate, using a meta-analysis, all evidence concerning the use of dabigatran versus warfarin in catheter ablation for AF. The main objectives of this study were: (1) evaluate the efficacy and safety of dabigatran compared to warfarin; (2) determine whether there were significant differences between the $110 \mathrm{mg}$ twice daily and $150 \mathrm{mg}$ twice daily dosages of dabigatran; and (3) study the management of periprocedural bridging anticoagulation.

\section{METHODS}

Data sources and search strategy

We performed a search on Medline (via Ovid and PubMed), Embase, and Cochrane (from inception to 13 April 2013) databases using the following search string: "atrial fibrillation" AND "ablation" AND "dabigatran".

The reference lists of the accessed full text articles were further researched for sources of potential information relevant to this analysis. Experts in the field were contacted to ensure that all important studies in this area were covered. Abstract authors in 
congresses were also contacted by email in order to retrieve additional information. The databases were reassessed before the completion of the manuscript to find if any of the included abstracts had been published as full text meanwhile.

\section{Inclusion and exclusion criteria}

The population, intervention, comparison, and outcome approach was used for conducting the meta-analysis. ${ }^{10}$ The population of interest included patients with AF. The intervention was catheter ablation of $\mathrm{AF}$ and the associated periprocedural anticoagulation. Comparisons were performed between the following groups: warfarin versus dabigatran; dabigatran $110 \mathrm{mg}$ twice daily vs $150 \mathrm{mg}$ twice daily. The primary outcomes were: major bleeding, thromboembolism, and all cause mortality. The presence of minor bleeding, when reported, was also assessed.

Only controlled trials (full text articles or conference abstracts) of patients undergoing catheter ablation of $\mathrm{AF}$ and treated either with warfarin or dabigatran before the procedure were selected.

The minimum necessary follow-up for study inclusion was until discharge after the procedure. Additionally, to be included, studies needed to provide information on assessment of all three major clinical outcome parameters: major bleeding, thromboembolism, and death.

The following exclusion criteria were defined: trials with no comparator (ie, warfarin), namely observational studies including only patients treated with dabigatran; starting of dabigatran treatment only after catheter ablation; and evaluation of efficacy restricted to laboratory or imaging end points.

To ensure that trials met the prespecified inclusion criteria, search results were reviewed by three investigators (RP, AB, BC), who needed to be in agreement for study selection.

Data extraction and presentation for the preparation of this manuscript followed the recommendations of the PRISMA group. ${ }^{11}$

Study quality was formally evaluated using the Delphi Consensus criteria for randomised controlled trials ${ }^{12}$ and a modified Newcastle-Ottawa Quality Assessment Scale for Case Control Studies ${ }^{13}$ by three reviewers (RP, AB, BC). An agreement, between the three reviewers was mandatory for the final classification of studies.

\section{End point definition for meta-analysis}

Classification of bleeding was based on previous reports and suggested recommendations. ${ }^{14}$ The following end points were defined for the generation of forest plots on meta-analysis.

The primary end points were:

1. All cause periprocedural death

2. Thromboembolism-a composite of stroke, transient ischaemic attack (TIA), systemic or pulmonary embolism. Asymptomatic cerebral embolism detected on routine or protocol MRI or CT (ie, imaging end points with no clinical manifestation) was not included in the analysis.

3. Major bleeding-comprising cardiac tamponade, bleeding necessitating intervention or transfusion, massive haemoptysis, haemothorax, retroperitoneal bleeding or any other lifethreatening bleed leading to prolongation of hospitalisation.

Secondary end point was:

1. Minor bleeding-defined as puncture site bleeding, thigh ecchymosis or haematoma, pericardial effusion with no haemodynamic compromise, minor gastrointestinal bleeding, epistaxis or any bleeding treated conservatively with no need for transfusion, surgery or prolonged hospitalisation.

\section{Assessment of periprocedural bridging of anticoagulation}

The following data were extracted for assessing periprocedural bridging anticoagulation: periprocedural warfarin regimen (uninterrupted drug or discontinuation); timing of dabigatran interruption and restart; target activated clotting time (ACT).

\section{Statistical analysis}

Data were pooled using random effects, according to the MantelHaenszel model, through Review Manager (RevMan), V.5.1. (Copenhagen: The Nordic Cochrane Centre, The Cochrane Collaboration, 2011). The measurement of treatment effect was performed using risk ratios (RR) and 95\% CIs. Pairwise comparisons were performed for all end points between patients treated with dabigatran and warfarin, and a separate sub-analysis (sensitivity analysis) was performed among patients treated with dabigatran, according to the dosage mentioned in the trials $(150 \mathrm{mg}$ twice daily vs $110 \mathrm{mg}$ twice daily). Sensitivity analysis was also performed restricting the analysis of data to: trials whose patients were treated with uninterrupted warfarin, prospective studies, investigations published as full text articles, studies whose follow-up was at least 30 days, and studies in which five of the nine items on the Delphi Consensus criteria for randomised controlled trials and a modified Newcastle-Ottawa Quality Assessment Scale for casecontrol studies were deemed satisfactory.

Statistical heterogeneity on each outcome of interest was assessed and quantified using the Cochran Q test and the $\mathrm{I}^{2}$ statistic, respectively. The presence of publication bias was evaluated by the use of funnel plots.

\section{RESULTS}

\section{Search results}

Overall, 228 entries were retrieved for title and abstract analysis. Of these, 169 were excluded as they did not meet inclusion criteria for the meta-analysis. The remaining 59 results were carefully screened, and after analysis of the full text (in the case of journal articles), only 14 studies (nine full text papers ${ }^{9-22}$ and five conference abstracts ${ }^{23-27}$ ) were deemed adequate for our review's purpose. The stepwise selection process is illustrated in figure 1 . There was a good agreement between investigators on the inclusion of the selected trials.

Baseline data and the design of selected trials are summarised in tables 1 and 2. The final population for this meta-analysis was composed of 4782 patients (1823 treated with dabigatran and 2959 with warfarin). Table 3 illustrates the assessment of the included studies through the Delphi criteria for randomised studies and the Newcastle-Ottawa Scale for non-randomised case-control studies.

The observed $\mathrm{I}^{2}$ values showed a low to moderate heterogeneity in the main end point comparisons $\left(\mathrm{I}^{2}=0\right.$ for stroke and $41 \%$ for major bleeding).

\section{Efficacy and safety of dabigatran versus warfarin}

The main efficacy and safety outcomes (prevention of embolism and major bleeding) observed in the comparison between dabigatran and warfarin are displayed in figure 2 and table 4 . The funnel plot to ascertain publication bias is shown in figure 3 .

\section{Death and thromboembolism}

No deaths were observed in any of the intervention groups. Thromboembolic events were reported in only $0.31 \%$ of patients (15 out of 4782) and no significant differences were found between dabigatran and warfarin: $0.55 \%$ vs $0.17 \%$, respectively $(\mathrm{RR}=1.78,95 \% \mathrm{CI} 0.66$ to $4.80 ; \mathrm{p}=0.26)$. 


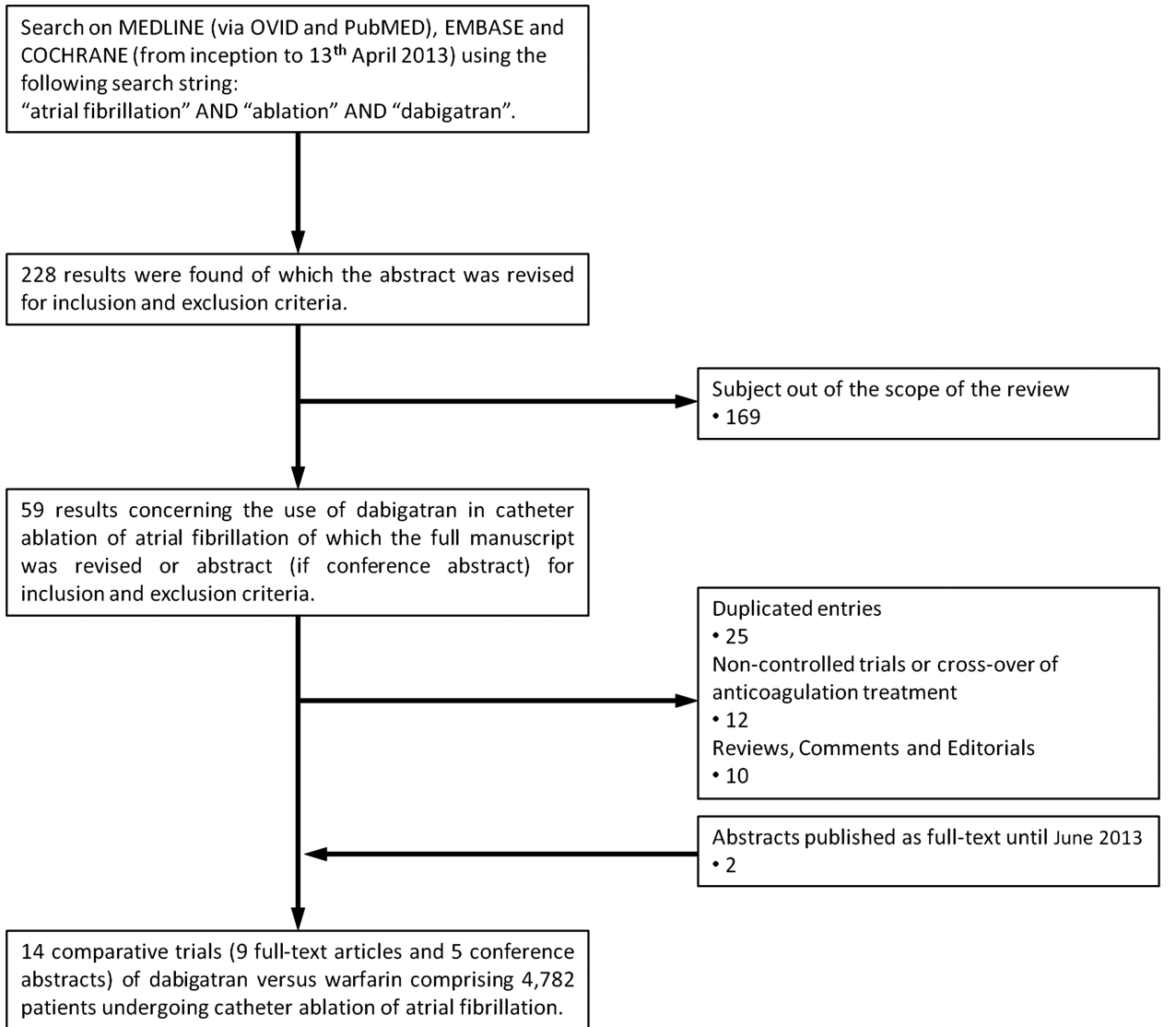

Figure 1 Flowchart diagram illustrating study selection.

\section{Major bleeding}

Among all 14 studies, 67 events (accounting for an overall $1.40 \%$ incidence) of major bleeding were reported, with no significant differences observed between the two treatment arms (1.48\% dabigatran vs $1.35 \%$ warfarin; $\mathrm{RR}=1.07$, 95\% CI 0.51 to $2.26 ; \mathrm{p}=0.86$ ).

\section{Minor bleeding}

Information on minor bleeding was not reported in one of the selected studies. ${ }^{24}$ In the remaining, comprising 4572 participants, 210 minor bleeding events (overall incidence 4.59\%; $3.35 \%$ dabigatran vs $5.40 \%$ warfarin) were reported, with a relative risk reduction of $35 \%(95 \%$ CI 7 to $55 \%$; $=0.02)$ in favour of dabigatran (figure 4).

\section{Sensitivity analysis}

Sensitivity analysis confirmed the lack of interference of uninterrupted warfarin, prospective studies, full text articles, studies whose follow-up was at least 30 days, and higher methodological quality studies (figures 5-7) in the overall results concerning the main end points: stroke and thromboembolism and major bleeding. However, as regards minor bleeding these analyses suggested a lack of significant differences.

\section{Effect of dabigatran dosage: $150 \mathrm{mg}$ twice daily vs $110 \mathrm{mg}$ twice daily}

Information on the dose of dabigatran used was available for 1392 patients (155 with $110 \mathrm{mg}$ twice daily and 1237 with $150 \mathrm{mg}$ twice daily). No significant differences were found between the two dosage groups $(110 \mathrm{mg}$ twice daily vs $150 \mathrm{mg}$ twice daily, respectively) regarding major bleeding (0\% vs
$1.62 \% ; \mathrm{RR}=0.19,95 \% \mathrm{CI} 0.01$ to $3.18 ; \mathrm{p}=0.25)$ and thromboembolism ( $0 \%$ vs $0.40 \%$; $\mathrm{RR}=0.72,95 \%$ CI 0.04 to $12.98 ; \mathrm{p}=0.82)$. Concerning minor bleeding, more events were observed with the $110 \mathrm{mg}$ twice daily dosage: $9.03 \%$ vs $2.51 \%$ $(\mathrm{RR}=3.60,95 \%$ CI 1.96 to $6.62 ; \mathrm{p}<0.0001)$.

\section{Management of periprocedural anticoagulation}

The different periprocedural regimens used in patients treated with dabigatran are displayed in table 2 . The timing of the first withheld dose ranged from the morning of procedure (in four studies) to $48 \mathrm{~h}$ before; the time interval for restarting ranged from $3-4 \mathrm{~h}$ (in six studies) to $24 \mathrm{~h}$ after ablation. In one study, uninterrupted dabigatran was used. ${ }^{19}$

One third of all strokes or TIAs were associated with suspension of dabigatran for $\geq 24 \mathrm{~h}$ before procedure (one stroke in Haines $e t a l^{16}$ and one stroke and one TIA in Rowley $e t a l^{27}$ ). Additionally, of all stroke and TIA in patients treated with dabigatran, $50 \%$ was related to a later timing ( $\geq 12 \mathrm{~h}$ ) for restarting the drug post-procedure (two strokes and two TIAs ${ }^{16}{ }^{27}$ ). On the other hand, a higher incidence of major bleeding was reported by Lakkireddy et $a l,{ }^{9}$ where dabigatran was stopped only in the morning of the procedure and restarted $3 \mathrm{~h}$ postprocedure. Albeit suggesting a similar safety and efficacy profile, with non-inferiority versus warfarin, data concerning uninterrupted dabigatran results exclusively from one trial. ${ }^{19}$

Among the patients of the warfarin group, safety and efficacy outcomes were similar irrespective of whether warfarin was continued or interrupted.

In addition, target ACT during procedure was 300-350 s in most studies, $>350 \mathrm{~s}$ in five, ${ }^{9} 15^{19-21}$ and $250-300 \mathrm{~s}$ in one. ${ }^{26}$ A higher value for target ACT $^{9} 15{ }^{19-21}$ was not associated with 
Table 1 Baseline characteristics of included studies

\begin{tabular}{|c|c|c|c|c|c|c|c|c|c|c|c|c|c|c|}
\hline & $\begin{array}{l}\text { Bassiouny } \\
\text { et } a l^{15}\end{array}$ & $\begin{array}{l}\text { Bernard } \\
\text { et } a l^{23}\end{array}$ & Haines et al ${ }^{16}$ & Ichiki et $\left.a\right|^{24}$ & $\begin{array}{l}\text { Kaseno } \\
\text { et } a l^{17}\end{array}$ & Kim et $a l^{18}$ & $\begin{array}{l}\text { Lakkireddy } \\
\text { et } \text { al }^{9}\end{array}$ & $\begin{array}{l}\text { Maddox } \\
\text { et al. }{ }^{19}\end{array}$ & $\begin{array}{l}\text { Mendoza } \\
\text { et } a l^{25}\end{array}$ & Nin et $a l^{20}$ & Pavaci et $a l^{26}$ & $\begin{array}{l}\text { Rowley } \\
\text { et } a l^{27}\end{array}$ & $\begin{array}{l}\text { Snipelisky } \\
\text { et } a^{21}\end{array}$ & Yamaji et $a^{22}$ \\
\hline Source type & $\begin{array}{l}\text { Journal } \\
\text { article }\end{array}$ & $\begin{array}{l}\text { Conference } \\
\text { abstract }\end{array}$ & Journal article & $\begin{array}{l}\text { Conference } \\
\text { abstract }\end{array}$ & $\begin{array}{l}\text { Journal } \\
\text { article }\end{array}$ & Journal article & Journal article & Journal article & $\begin{array}{l}\text { Conference } \\
\text { abstract }\end{array}$ & $\begin{array}{l}\text { Journal } \\
\text { article }\end{array}$ & $\begin{array}{l}\text { Conference } \\
\text { abstract }\end{array}$ & $\begin{array}{l}\text { Conference } \\
\text { abstract }\end{array}$ & $\begin{array}{l}\text { Journal } \\
\text { article }\end{array}$ & Journal article \\
\hline Study design & $\begin{array}{l}\text { Prospective, } \\
\text { monocentric } \\
\text { registry }\end{array}$ & Retrospective & $\begin{array}{l}\text { Multicentric, } \\
\text { retrospective, } \\
\text { case-match } \\
\text { analysis }\end{array}$ & $\begin{array}{l}\text { Prospective, } \\
\text { non-randomised }\end{array}$ & Retrospective & Retrospective & $\begin{array}{l}\text { Multicentric, } \\
\text { prospective, } \\
\text { non-randomised }\end{array}$ & Retrospective & Retrospective & $\begin{array}{l}\text { Prospective, } \\
\text { randomised }\end{array}$ & $\begin{array}{l}\text { Retrospective, } \\
\text { case-match } \\
\text { analysis }\end{array}$ & Retrospective & Retrospective & $\begin{array}{l}\text { Retrospective, } \\
\text { non-randomised } \\
\text { and randomised }\end{array}$ \\
\hline Paroxysmal AF & $\begin{array}{l}57 \% \text { D } \\
55 \% \text { W }\end{array}$ & $\begin{array}{l}46 \% \text { D } \\
50 \% \text { W }\end{array}$ & $\begin{array}{l}53 \% \mathrm{D} \\
48 \% \mathrm{~W}\end{array}$ & $53 \%$ & $\begin{array}{l}83 \% \mathrm{D} \\
55 \% \mathrm{~W}\end{array}$ & $\begin{array}{l}53 \% \mathrm{D} \\
48 \% \mathrm{~W}\end{array}$ & $\begin{array}{l}57 \% \text { D } \\
57 \% \text { W }\end{array}$ & $\begin{array}{l}63 \% \mathrm{D} \\
57 \% \mathrm{~W}\end{array}$ & NA & $\begin{array}{l}76 \% \mathrm{D} \\
71 \% \mathrm{~W}\end{array}$ & NA & NA & $\begin{array}{l}68 \% \mathrm{D} \\
46 \% \mathrm{~W}\end{array}$ & $\begin{array}{l}65 \% \mathrm{D} \\
62 \% \mathrm{~W}\end{array}$ \\
\hline Age & $\begin{array}{l}58.6 \mathrm{D} \\
62.7 \mathrm{~W}\end{array}$ & $\begin{array}{l}62.7 \mathrm{D} \\
67.3 \mathrm{~W}\end{array}$ & $\begin{array}{l}60.2 \mathrm{D} \\
59.7 \mathrm{~W}\end{array}$ & n.s. & $\begin{array}{l}59 \mathrm{D} \\
62 \mathrm{~W}\end{array}$ & $\begin{array}{l}61 \mathrm{D} \\
61 \mathrm{~W}\end{array}$ & $\begin{array}{l}60.4 \mathrm{D} \\
60.3 \mathrm{~W}\end{array}$ & $\begin{array}{l}62.3 \mathrm{D} \\
62.5 \mathrm{~W}\end{array}$ & $\begin{array}{l}62.9 \mathrm{D} \\
64.0 \mathrm{~W}\end{array}$ & $\begin{array}{l}61 \mathrm{D} \\
61 \mathrm{~W}\end{array}$ & $\begin{array}{l}\text { NA } \\
\text { Case-matched }\end{array}$ & $\begin{array}{l}63 \pm 10 \\
\text { similar }\end{array}$ & $\begin{array}{l}60.6 \mathrm{D} \\
64.6 \mathrm{~W}\end{array}$ & $\begin{array}{l}60 \mathrm{D} \\
61 \mathrm{~W}\end{array}$ \\
\hline q Gender & $\begin{array}{l}25 \% \mathrm{D} \\
26.6 \% \mathrm{~W}\end{array}$ & NA & $\begin{array}{l}26 \% \mathrm{D} \\
31 \% \mathrm{~W}\end{array}$ & NA & $\begin{array}{l}25 \% \mathrm{D} \\
21 \% \mathrm{~W}\end{array}$ & $\begin{array}{l}20 \% \mathrm{D} \\
26 \% \mathrm{~W}\end{array}$ & $\begin{array}{l}21 \% \mathrm{D} \\
21 \% \mathrm{~W}\end{array}$ & $\begin{array}{l}24 \% \mathrm{D} \\
33 \% \mathrm{~W}\end{array}$ & $\begin{array}{l}10 \% \mathrm{D} \\
12 \% \mathrm{~W}\end{array}$ & $\begin{array}{l}16 \% \mathrm{D} \\
20 \% \mathrm{~W}\end{array}$ & $\begin{array}{l}\text { NA } \\
\text { Case-matched }\end{array}$ & NA & $\begin{array}{l}19.4 \% \mathrm{D} \\
25.6 \% \mathrm{~W}\end{array}$ & $\begin{array}{l}25 \% \mathrm{D} \\
24 \% \mathrm{~W}\end{array}$ \\
\hline $\begin{array}{l}\text { Estimated } \\
\text { thrombo-embolic } \\
\text { risk }\end{array}$ & $\begin{array}{l}\text { CHADS }_{2} \\
\text { Score }=0 \\
42.6 \% \text { D } \\
29.1 \% \text { W } \\
\text { Score }=1 \\
37.0 \% \text { D } \\
41.6 \% \text { W } \\
\text { Score } \geq 2 \\
20.5 \% \text { D } \\
29.4 \% \text { W }\end{array}$ & $\begin{array}{l}\mathrm{CHA}_{2} \mathrm{DS}_{2}-\mathrm{VASC} \\
2.00 \mathrm{D} \\
2.68 \mathrm{~W}\end{array}$ & $\begin{array}{l}\mathrm{CHA}_{2} \mathrm{DS}_{2} \text {-VASC } \\
1.6 \pm 1.3 \mathrm{D} \\
1.9 \pm 1.4 \mathrm{~W}\end{array}$ & $\begin{array}{l}\text { Values NA } \\
\text { ns differences } \\
\text { between } \\
\text { treatment } \\
\text { groups }\end{array}$ & $\begin{array}{l}\text { CHADS }_{2} \\
\text { Score }=0 \\
61 \% \text { D 45\% } \\
\text { W } \\
\text { Score }=1 \\
31 \% \text { D } 41 \% \\
\text { W } \\
\text { Score } \geq 2 \\
8 \% \text { D } 14 \% \\
\text { W }\end{array}$ & $\begin{array}{l}\mathrm{CHA}_{2} \mathrm{DS}_{2} \text {-VASC } \\
1.6 \pm 1.3 \mathrm{D} \\
1.7 \pm 1.3 \mathrm{~W}\end{array}$ & $\begin{array}{l}\mathrm{CHADS}_{2} \\
\text { Score=0 } \\
35 \% \text { D 40\% W } \\
\text { Score }=1 \\
43 \% \text { D 41\% W } \\
\text { Score } \geq 2 \\
23 \% \text { D 19\% W } \\
\mathrm{CHA}_{2} \mathrm{DS}_{2} \text {-VASC } \\
1.6 \pm 1.4 \mathrm{D} \\
1.5 \pm 1.3 \mathrm{~W}\end{array}$ & $\begin{array}{l}\mathrm{CHADS}_{2} \\
0.92 \pm 0.88 \mathrm{D} \\
0.92 \pm 0.85 \mathrm{~W} \\
\mathrm{CHA}_{2} \mathrm{DS}_{2}-\mathrm{VASC} \\
1.73 \pm 1.45 \mathrm{D} \\
1.69 \pm 1.33 \mathrm{~W}\end{array}$ & $\begin{array}{l}\text { CHADS }_{2} \\
1.32 \mathrm{D} \\
1.29 \mathrm{~W}\end{array}$ & $\begin{array}{l}\text { CHADS }_{2} \\
\text { Score } \leq 1 \\
82 \% \text { D } 80 \% \\
\text { W } \\
\text { Score=1 } \\
11 \% \text { D } 13 \% \\
\text { W } \\
\text { Score } \geq 2 \\
4 \% \text { D } 11 \% \\
\text { W }\end{array}$ & NA & $\begin{array}{l}\mathrm{CHADS}_{2} \\
1.3 \pm 1\end{array}$ & $\begin{array}{l}\mathrm{CHADS}_{2} \\
0.80 \mathrm{D} \\
1.16 \mathrm{~W}\end{array}$ & $\begin{array}{l}\mathrm{CHADS}_{2} \\
\text { Score }=0 \\
36 \% \text { D 33\% W } \\
\text { Score }=1 \\
40 \% \text { D } 38 \% \text { W } \\
\text { Score } \geq 2 \\
24 \% \text { D 29\% W } \\
\text { CHA }_{2} \text { DS }_{2} \text {-VASC } \\
1.8 \pm 1.6 \text { D } \\
1.7 \pm 1.6 \mathrm{~W}\end{array}$ \\
\hline $\begin{array}{l}\text { Estimated } \\
\text { bleeding risk }\end{array}$ & NA & NA & NA & NA & $\begin{array}{l}\text { HAS-BLED } \\
0.5 \pm 0.7 \mathrm{D} \\
0.6 \pm 0.6 \mathrm{~W}\end{array}$ & $\begin{array}{l}\text { HAS-BLED } \\
1.0 \pm 0.9 \mathrm{D} \\
1.1 \pm 0.9 \mathrm{~W}\end{array}$ & $\begin{array}{l}\text { HAS-BLED } \\
1.2 \pm 0.9 \mathrm{D} \\
1.1 \pm 0.9 \mathrm{~W}\end{array}$ & NA & $\begin{array}{l}\text { HAS-BLED } \\
1.47 \mathrm{D} \\
1.63 \mathrm{~W}\end{array}$ & NA & NA & NA & NA & NA \\
\hline $\begin{array}{l}\text { LV ejection } \\
\text { fraction }\end{array}$ & $\begin{array}{l}55 \% \text { D } \\
55 \% \text { W }\end{array}$ & $\begin{array}{l}60 \% \text { D } \\
58 \% \text { W }\end{array}$ & $\begin{array}{l}56 \% \mathrm{D} \\
57 \% \mathrm{~W}\end{array}$ & NA & $\begin{array}{l}64 \% \mathrm{D} \\
63 \% \mathrm{~W}\end{array}$ & $\begin{array}{l}58 \% \mathrm{D} \\
57 \% \mathrm{~W}\end{array}$ & $\begin{array}{l}56 \% \mathrm{D} \\
56 \% \mathrm{~W}\end{array}$ & $\begin{array}{l}53 \% \mathrm{D} \\
54 \% \mathrm{~W}\end{array}$ & NA & $\begin{array}{l}61 \% \mathrm{D} \\
62 \% \mathrm{~W}\end{array}$ & NA & NA & NA & $\begin{array}{l}60 \% \mathrm{D} \\
60 \% \mathrm{~W}\end{array}$ \\
\hline $\begin{array}{l}\text { CKD/kidney } \\
\text { function }\end{array}$ & $\begin{array}{l}\text { Creat } \\
0.9 \mathrm{mg} / \mathrm{dL} \mathrm{D} \\
0.9 \mathrm{mg} / \mathrm{dL} \mathrm{W}\end{array}$ & $\begin{array}{l}\text { Creat } \\
1.0 \mathrm{mg} / \mathrm{dL} \mathrm{D} \\
1.3 \mathrm{mg} / \mathrm{dL} \mathrm{W}\end{array}$ & $\begin{array}{l}\text { Creat clearance } \\
93 \mathrm{~m} / / \min D \\
93 \mathrm{~m} / / \mathrm{min} \mathrm{W}\end{array}$ & NA & $\begin{array}{l}\text { Creat } \\
\text { clearance } \\
97 \mathrm{ml} / \mathrm{min} \mathrm{D} \\
98 \mathrm{ml} / \mathrm{min} \mathrm{W}\end{array}$ & $\begin{array}{l}\text { Creat } \\
0.9 \mathrm{mg} / \mathrm{dL} \text { D } \\
0.9 \mathrm{mg} / \mathrm{dLW}\end{array}$ & $\begin{array}{l}\text { CKD } \\
1 \% D \\
2 \% W\end{array}$ & $\begin{array}{l}\text { Creat } \\
1.0 \mathrm{mg} / \mathrm{dL} \mathrm{D} \\
1.1 \mathrm{mg} / \mathrm{dL} \mathrm{W}\end{array}$ & NA & $\begin{array}{l}\text { Creat } \\
0.9 \mathrm{mg} / \mathrm{dL} \text { D } \\
0.9 \mathrm{mg} / \mathrm{dL} \mathrm{W}\end{array}$ & NA & NA & NA & $\begin{array}{l}\text { Exclusion of } \\
\text { patients with } \\
\text { renal clearance } \\
<30 \mathrm{~mL} / \mathrm{min}\end{array}$ \\
\hline
\end{tabular}


Table 2 Description of periprocedural anticoagulation

\begin{tabular}{|c|c|c|c|c|c|c|c|c|c|c|c|c|c|c|}
\hline & $\begin{array}{l}\text { Bassiouny } \\
\text { et } a^{15}\end{array}$ & $\begin{array}{l}\text { Bernard } \\
\text { et } a l^{23}\end{array}$ & Haines et al ${ }^{16}$ & Ichiki et $\left.a\right|^{24}$ & $\begin{array}{l}\text { Kaseno } \\
\text { et } a 1^{17}\end{array}$ & Kim et $a l^{18}$ & $\begin{array}{l}\text { Lakkireddy } \\
\text { et } a l^{9}\end{array}$ & $\begin{array}{l}\text { Maddox } \\
\text { et } a^{19}\end{array}$ & $\begin{array}{l}\text { Mendoza } \\
\text { et } a l^{25}\end{array}$ & Nin et $a^{20}$ & $\begin{array}{l}\text { Pavaci } \\
\text { et }\left.a\right|^{26}\end{array}$ & $\begin{array}{l}\text { Rowley } \\
\text { et }\left.a\right|^{27}\end{array}$ & $\begin{array}{l}\text { Snipelisky } \\
\text { et al. }{ }^{21}\end{array}$ & Yamaji et $a l^{22}$ \\
\hline $\begin{array}{l}\text { Patients } \\
\text { treated with } \\
\text { dabigatran }\end{array}$ & 376 & 155 & 222 & 30 & 110 & 191 & 145 & 212 & 60 & 45 & 27 & 113 & 31 & 106 \\
\hline $\begin{array}{l}\text { Dabigatran } \\
\text { dosage }\end{array}$ & $\begin{array}{l}150 \mathrm{mg} \text { twice } \\
\text { daily }\end{array}$ & NA & $\begin{array}{l}150 \mathrm{mg} \text { twice } \\
\text { daily* }\end{array}$ & NA & $\begin{array}{l}110 \mathrm{mg} \text { twice } \\
\text { daily }\end{array}$ & $\begin{array}{l}150 \mathrm{mg} \text { twice } \\
\text { daily }\end{array}$ & $\begin{array}{l}150 \mathrm{mg} \text { twice } \\
\text { daily }\end{array}$ & $\begin{array}{l}150 \mathrm{mg} \text { twice } \\
\text { daily }\end{array}$ & $\begin{array}{l}150 \mathrm{mg} \text { twice } \\
\text { daily }\end{array}$ & $\begin{array}{l}110 \mathrm{mg} \text { twice } \\
\text { daily }\end{array}$ & NA & NA & $\begin{array}{l}150 \mathrm{mg} \\
\text { twice daily }\end{array}$ & $\begin{array}{l}110 \mathrm{mg} \text { twice } \\
\text { daily in } 36 \\
150 \mathrm{mg} \text { twice } \\
\text { daily in } 70\end{array}$ \\
\hline $\begin{array}{l}\text { Timing of } \\
\text { first held } \\
\text { dose of } \\
\text { dabigatrain }\end{array}$ & $\begin{array}{l}\text { Morning of } \\
\text { the day of the } \\
\text { procedure or } \\
\text { the night } \\
\text { before }\end{array}$ & $24 \mathrm{~h}$ & $\begin{array}{l}\leq 12 \mathrm{~h} \text { in } 35 \\
12-24 \mathrm{~h} \text { in } 29 \\
24-48 \mathrm{~h} \text { in } 102 \\
>48 \mathrm{~h} \text { in } 18 \\
\text { Unknown in } 18\end{array}$ & $\begin{array}{l}\text { Morning of } \\
\text { procedure }\end{array}$ & $\begin{array}{l}\text { Morning of } \\
\text { procedure }\end{array}$ & $\begin{array}{l}\text { Night before } \\
\text { procedure }\end{array}$ & $\begin{array}{l}\text { Morning of } \\
\text { procedure }\end{array}$ & Uninterrupted & $\begin{array}{l}\text { Morning of } \\
\text { procedure }\end{array}$ & $\begin{array}{l}\text { Morning of } \\
\text { the day } \\
\text { before } \\
\text { procedure }\end{array}$ & NA & $\begin{array}{l}\text { Day } \\
\text { before } \\
\text { procedure }\end{array}$ & $\begin{array}{l}\text { Morning of } \\
\text { procedure }\end{array}$ & $\begin{array}{l}\text { Morning of the } \\
\text { day of the } \\
\text { procedure }\end{array}$ \\
\hline $\begin{array}{l}\text { Time } \\
\text { interval for } \\
\text { restarting } \\
\text { after } \\
\text { procedure }\end{array}$ & $\begin{array}{l}\text { After arousal } \\
\text { from } \\
\text { anesthesia/ } \\
\text { sedation }\end{array}$ & Within $24 \mathrm{~h}$ & $\begin{array}{l}\leq 6 \mathrm{~h} \text { in } 116 \\
6-24 \mathrm{~h} \text { in } 83 \\
>24 \mathrm{~h} \text { in } 1 \\
\text { Unknown in } 2\end{array}$ & NA & $\begin{array}{l}\text { Morning after } \\
\text { procedure }\end{array}$ & $4 \mathrm{~h}$ & $3 \mathrm{~h}$ & Uninterrupted & $\begin{array}{l}\text { Immediately } \\
\text { after sheath } \\
\text { removal } \\
\text { (144.3 } \mathrm{min} \text { ) }\end{array}$ & $\begin{array}{l}4 \mathrm{~h} \text { after } \\
\text { haemostasis } \\
\text { at the } \\
\text { puncture site }\end{array}$ & NA & $\begin{array}{l}>24 \mathrm{~h} \\
\text { (day } \\
\text { following) }\end{array}$ & $\begin{array}{l}\text { Evening of } \\
\text { procedure }\end{array}$ & $\begin{array}{l}\text { Single dose, } \\
3 \mathrm{~h} \text { after the } \\
\text { completion of } \\
\text { the procedure }\end{array}$ \\
\hline $\begin{array}{l}\text { Patients } \\
\text { treated with } \\
\text { warfarin }\end{array}$ & 623 & 44 & 222 & 180 & 101 & 572 & 145 & 251 & 58 & 45 & 27 & 169 & 125 & 397 \\
\hline $\begin{array}{l}\text { Timing of } \\
\text { first held } \\
\text { dose of } \\
\text { warfarin }\end{array}$ & Uninterrupted & Uninterrupted & $\begin{array}{l}\leq 12 \mathrm{~h} \text { in } 74 \\
12-24 \mathrm{~h} \text { in } 88 \\
24-48 \mathrm{~h} \text { in } 15 \\
>48 \mathrm{~h} \text { in } 25\end{array}$ & Uninterrupted & Uninterrupted & Uninterrupted & Uninterrupted & Uninterrupted & Uninterrupted & $\begin{array}{l}\text { Morning of } \\
\text { the day } \\
\text { before } \\
\text { procedure }\end{array}$ & Uninterrupted & $\begin{array}{l}\text { Day } \\
\text { before } \\
\text { procedure }\end{array}$ & $\begin{array}{l}\text { Evening } \\
\text { prior to the } \\
\text { procedure }\end{array}$ & $\begin{array}{l}\text { Uninterrupted } \\
\text { in } 203 \text { patients. } \\
\text { Interrupted } \\
48 \mathrm{~h} \text { before in } \\
194\end{array}$ \\
\hline $\begin{array}{l}\text { Timing for } \\
\text { restarting } \\
\text { warfarin }\end{array}$ & Uninterrupted & Uninterrupted & $\begin{array}{l}\text { Day of the } \\
\text { procedure }\end{array}$ & Uninterrupted & Uninterrupted & Uninterrupted & Uninterrupted & Uninterrupted & Uninterrupted & $\begin{array}{l}4 \mathrm{~h} \text { after } \\
\text { haemostasis } \\
\text { at the } \\
\text { puncture site }\end{array}$ & Uninterrupted & $\begin{array}{l}\text { Day after } \\
\text { procedure }\end{array}$ & $\begin{array}{l}\text { Evening of } \\
\text { procedure }\end{array}$ & $\begin{array}{l}\text { Day after } \\
\text { procedure in } \\
\text { patients who } \\
\text { stopped } \\
\text { warfarin }\end{array}$ \\
\hline $\begin{array}{l}\text { Target ACT } \\
\text { (seconds) } \\
\text { during } \\
\text { procedure }\end{array}$ & $350-450$ & NA & $\begin{array}{l}300-350 \text { at } \\
\text { least }\end{array}$ & NA & $300-350$ & $300-350$ & $300-400$ & $>350-400$ & $300-350$ & $300-400$ & $250-300$ & NA & $>350$ & $300-350$ \\
\hline $\begin{array}{l}\text { Follow-up } \\
\text { duration }\end{array}$ & 1 month & 1 month & $\begin{array}{l}\text { Periprocedural/ } \\
\text { hospital } \\
\text { discharge }\end{array}$ & NA & $\begin{array}{l}\text { At least } \\
2 \text { months }\end{array}$ & 3 months & 30 days & $\begin{array}{l}\text { Periprocedural/ } \\
\text { hospital } \\
\text { discharge }\end{array}$ & 1 month & 2 weeks & NA & 30 days & 1 week & 3 months \\
\hline
\end{tabular}


Table 3 Assessment of the quality of included studies: Delphi criteria for randomised studies and Newcastle-Ottawa Scale for non-randomised case-controls studies

\begin{tabular}{|c|c|c|}
\hline Study & Assessment & Classification* \\
\hline $\begin{array}{l}\text { Bassiouny } \\
\text { et }\left.a\right|^{15}\end{array}$ & $\begin{array}{l}\text { Adequate case definition; consecutive series of cases; adequate information concerning the selection and definition of controls; } \\
\text { groups controlled for baseline characteristics; ascertainment of outpatient exposure to anticoagulants based on medical records } \\
\text { both for cases and controls, and laboratory data for warfarin; patients not blinded to case-control status. }\end{array}$ & Attributable stars: 7 \\
\hline Bernard et $a l^{23}$ & $\begin{array}{l}\text { Adequate case definition; consecutive series of cases; controls were slightly older ( } 3 \text { years in average), had higher creatinine } \\
\left.\text { values ( } 1.3 \text { vs } 1.0 \mathrm{mg} / \mathrm{dL}) \text { and a higher } \mathrm{CHA}_{2} \mathrm{DS}_{2} \text {-VASc ( } 2.68 \text { vs } 2.00\right) \text {; information concerning the selection and definition of } \\
\text { controls. }\end{array}$ & Attributable stars: 3 \\
\hline Haines et $a l^{16}$ & $\begin{array}{l}\text { Adequate case definition; consecutive series of cases; hospital controls, obtained by computer list generation; groups controlled } \\
\text { for most baseline characteristics, except for slight differences in congestive heart failure, more prevalent in the dabigatran group, } \\
\text { and previous stroke, more prevalent in the warfarin group; a higher utilisation of concomitant aspirin was observed in dabigatran } \\
\text { patients and heparin/low molecular weight heparin was more used in the warfarin treatment arm; ascertainment of outpatient } \\
\text { exposure to anticoagulants based on medical records both for cases and controls, and laboratory data for warfarin; patients not } \\
\text { blinded to case-control status. }\end{array}$ & Attributable stars: 6 \\
\hline Ichiki et $\left.a\right|^{24}$ & $\begin{array}{l}\text { Adequate case definition; consecutive series of cases; adequate information concerning the selection and definition of controls; } \\
\text { information concerning the selection and definition of controls. }\end{array}$ & Attributable stars: 4 \\
\hline Kaseno et $a l^{17}$ & $\begin{array}{l}\text { Adequate case definition; information concerning the selection and definition of controls; hospital controls; slight difference in } \\
\text { baseline age (warfarin patients } 3 \text { years older), larger left atrial diameter ( } 4 \mathrm{~mm} \text { difference in average) and higher prevalence of } \\
\text { persistent AF in the warfarin group, requiring additional substrate modification; ascertainment of outpatient exposure to } \\
\text { anticoagulants based on medical records both for cases and controls, and laboratory data for warfarin; patients not blinded to } \\
\text { case-control status. }\end{array}$ & Attributable stars: 4 \\
\hline Kim et $a l^{18}$ & $\begin{array}{l}\text { Adequate case definition; consecutive series of cases; adequate information concerning the selection and definition of controls; } \\
\text { groups controlled for baseline characteristics, except for previous stroke, that was slightly more prevalent in the warfarin group; } \\
\text { ascertainment of outpatient exposure to anticoagulants based on medical records both for cases and controls, and laboratory data } \\
\text { for warfarin; close monitoring of all patients during the } 3 \text { month follow-up period after the procedure; patients not blinded to } \\
\text { case-control status. }\end{array}$ & Attributable stars: 6 \\
\hline $\begin{array}{l}\text { Lakkireddy } \\
\text { et al }{ }^{9}\end{array}$ & $\begin{array}{l}\text { Adequate case definition; consecutive series of cases in the dabigatran treatment arm; controls matched for age, sex and type of } \\
\text { AF; groups controlled for baseline characteristics; ascertainment of outpatient exposure to anticoagulants based on medical } \\
\text { records both for cases and controls, and laboratory data for warfarin; patients not blinded to case-control status. }\end{array}$ & Attributable stars: 6 \\
\hline Maddox et al ${ }^{19}$ & $\begin{array}{l}\text { Adequate case definition; consecutive series of cases; adequate information concerning the selection and definition of controls; } \\
\text { groups controlled for most baseline characteristics, except for a slightly higher percentage of males in the dabigatran group; } \\
\text { ascertainment of outpatient exposure to anticoagulants based on medical records both for cases and controls, and laboratory data } \\
\text { for warfarin; patients not blinded to case-control status. }\end{array}$ & Attributable stars: 6 \\
\hline $\begin{array}{l}\text { Mendoza } \\
\text { et } a l^{25}\end{array}$ & $\begin{array}{l}\text { Adequate case definition; consecutive series of cases; hospital controls; information concerning the selection and definition of } \\
\text { controls. }\end{array}$ & Attributable stars: 4 \\
\hline Nin et $a l^{20}$ & $\begin{array}{l}\text { Randomisation performed; groups similar at baseline; eligibility criteria specified; point estimates and measures of variability } \\
\text { presented for outcome measures; analysis of end points performed on an intention-to-treat basis. }\end{array}$ & $\begin{array}{l}\text { Positive Delphi } \\
\text { criteria: } 5\end{array}$ \\
\hline Pavaci et $a l^{26}$ & $\begin{array}{l}\text { Adequate case definition; consecutive series of cases treated with dabigatran; case matched hospital controls considering age, } \\
\text { gender, body mass index, creatinine values, left atrium dimensions and type of arrhythmia; information concerning the selection } \\
\text { and definition of controls. }\end{array}$ & Attributable stars: 4 \\
\hline Rowley et $a l^{27}$ & $\begin{array}{l}\text { Adequate case definition; series of cases treated with dabigatran, no information if the patients were consecutive; no information } \\
\text { concerning the selection and definition of controls; similar age and } \mathrm{CHADS}_{2} \text { score among treatment groups. }\end{array}$ & Attributable stars: 3 \\
\hline $\begin{array}{l}\text { Snipelisky } \\
\text { et } a l^{21}\end{array}$ & $\begin{array}{l}\text { Adequate case definition; consecutive series of cases; adequate information concerning the selection and definition of controls; } \\
\text { groups controlled for baseline characteristics, except for higher prevalence of persistent AF in the warfarin group; ascertainment of } \\
\text { outpatient exposure to anticoagulants based on medical records both for cases and controls, and laboratory data for warfarin; } \\
\text { patients not blinded to case-control status; all patients had evidence for a completed medication profile with reconciliation. }\end{array}$ & Attributable stars: 7 \\
\hline Yamaji et $a l^{22}$ & $\begin{array}{l}\text { Adequate case definition; consecutive series of patients assigned to treatment groups in a non-randomised fashion in the first } \\
\text { part of the study and with randomisation to dabigatran or uninterrupted warfarin in the last } 212 \text { patients; adequate information } \\
\text { concerning the selection and definition of controls; groups controlled for baseline characteristics; ascertainment of outpatient } \\
\text { exposure to anticoagulants based on medical records both for cases and controls, and laboratory data for warfarin; close } \\
\text { monitoring of all patients during the 3-month follow-up period after the procedure; patients not blinded to case-control status. }\end{array}$ & Attributable stars: 7 \\
\hline
\end{tabular}

a lower incidence of thromboembolic events. Furthermore, in the investigation by Lakkireddy et $a l^{9}$ a higher rate of cardiac tamponade in dabigatran treated patients $(9 / 145)$ was observed. Importantly, a $3.7 \%$ rate of stroke was observed in the single study featuring a target ACT of 250-300 s. ${ }^{26}$ However, due to the low number of participants, the possibility of a false positive association cannot be excluded.

\section{DISCUSSION}

The low rate of thromboembolic complications and major bleeding seen with dabigatran (which was also similar to that observed with warfarin) seems to provide favourable support for its use, as an alternative to warfarin, in the setting of catheter ablation of AF. These results add important information on the use of dabigatran in this setting. They also serve to potentially alleviate some of the concerns raised by the rather unfavourable results in earlier studies $(2.1 \%$ stroke or TIA and $6.2 \%$ major bleeds), ${ }^{9}$ suggesting that dabigatran significantly increased the risk of bleeding or thromboembolic complications compared with uninterrupted warfarin therapy.

A very low rate of stroke and thromboembolic events was found in this meta-analysis among patients treated with dabigatran $(0.55 \%)$, similar to what has been found in a recent analysis of Medicare beneficiaries (0.6-0.9\%). ${ }^{28}$ A lower, but not significantly different, prevalence of embolism was found in the warfarin arm of this meta-analysis $(0.17 \%)$, composed mainly of 
A

\begin{tabular}{|c|c|c|c|c|c|c|c|c|}
\hline \multirow[b]{3}{*}{ Study or Subgroup } & \multicolumn{8}{|c|}{ Stroke and Thromboembolism } \\
\hline & \multicolumn{2}{|c|}{ Dabigatran } & \multicolumn{2}{|c|}{ Warfarin } & \multicolumn{2}{|c|}{ Risk Ratio } & \multirow{2}{*}{\multicolumn{2}{|c|}{$\begin{array}{c}\text { Risk Ratio } \\
\text { M-H, Random, } 95 \% \mathrm{Cl}\end{array}$}} \\
\hline & Events & Total & Events & Total & Weight & $\mathrm{M}-\mathrm{H}, \mathrm{Random}, 95 \% \mathrm{Cl}$ & & \\
\hline Bassiouny 2013 & 1 & 376 & 1 & 623 & $12.9 \%$ & $1.66[0.10,26.41]$ & & \\
\hline Bernard 2013 & 0 & 155 & 0 & 44 & & Not estimable & & \\
\hline Haines 2012 & 2 & 222 & 0 & 222 & $10.7 \%$ & $5.00[0.24,103.56]$ & & \\
\hline Ichiki 2012 & 0 & 30 & 0 & 180 & & Not estimable & & \\
\hline Kaseno 2012 & 0 & 110 & 0 & 101 & & Not estimable & & \\
\hline Kim 2012 & 0 & 191 & 0 & 572 & & Not estimable & & \\
\hline Lakkireddy 2012 & 3 & 145 & 0 & 145 & $11.3 \%$ & $7.00[0.36,134.32]$ & & \\
\hline Maddox 2013 & 1 & 212 & 0 & 251 & $9.7 \%$ & $3.55[0.15,86.67]$ & & \\
\hline Mendoza 2012 & 0 & 60 & 1 & 58 & $9.7 \%$ & $0.32[0.01,7.76]$ & & \\
\hline $\operatorname{Nin} 2012$ & 0 & 45 & 1 & 45 & $9.8 \%$ & $0.33[0.01,7.97]$ & & \\
\hline Pavaci 2012 & 1 & 27 & 0 & 27 & $9.9 \%$ & $3.00[0.13,70.53]$ & & \\
\hline Rowley 2012 & 2 & 113 & 2 & 169 & $26.0 \%$ & $1.50[0.21,10.46]$ & & \\
\hline Snipelsky 2012 & 0 & 31 & 0 & 125 & & Not estimable & & \\
\hline Yamaji 2012 & 0 & 106 & 0 & 397 & & Not estimable & & \\
\hline Total $(95 \% \mathrm{Cl})$ & & 1823 & & 2959 & $100.0 \%$ & $1.78[0.66,4.80]$ & & \\
\hline Total events & 10 & & 5 & & & & & \\
\hline $\begin{array}{l}\text { Heterogeneity: Tau² } \\
\text { Test for overall effect }\end{array}$ & $\begin{array}{l}0.00 ; \mathrm{Chi} \\
z=1.14\end{array}$ & $\begin{array}{l}=3.78 \\
=0.26\end{array}$ & df $d$ ) & $=0.80$ & ); $1^{2}=0 \%$ & & $\begin{array}{ll}0.01 & 0.1 \\
\text { Favours dabigatran } & 1\end{array}$ & $\begin{array}{l}10 \\
\text { Favours we }\end{array}$ \\
\hline
\end{tabular}

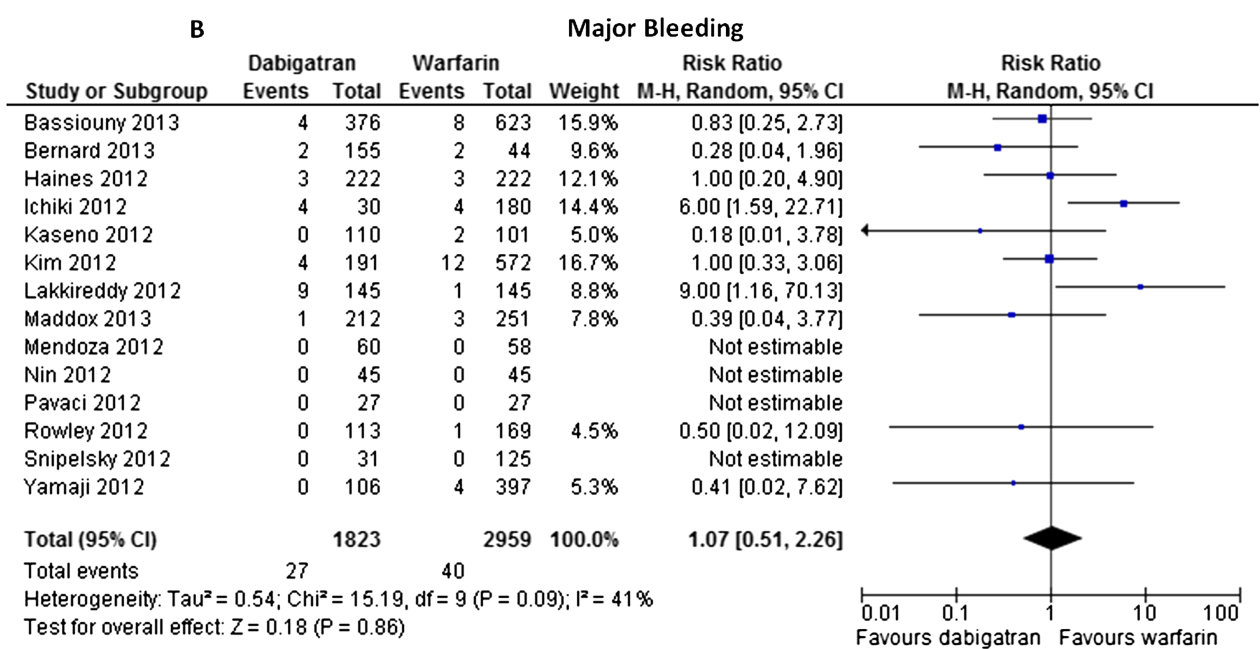

Figure 2 Main efficacy and safety outcomes of the comparison of dabigatran versus warfarin among patients with atrial fibrillation treated with catheter ablation. (A) Stroke and thromboembolism. (B) Major bleeding. M-H, Mantel-Haenszel.

patients with uninterrupted treatment. This value is similar to the one described by Hussein et $a l^{29}$ in patients with uninterrupted warfarin. Therefore, we think that despite showing similar results compared to warfarin in this meta-analysis (and presenting values for thromboembolic complications similar to what has been reported in large registries of patients using antivitamin $\mathrm{K}$ agents ${ }^{28}$ ), validation of these results in future randomised controlled trials with blinded analysis may be justified. Furthermore, the use of uninterrupted dabigatran may also merit assessment, taking into account the promising results of the recently available study with this treatment regimen. ${ }^{19}$

The proportion of patients referred for AF ablation who are on longstanding dabigatran therapy is progressively increasing. This is likely to pose new challenges for the practising cardiologist, including the question of performing the procedure without discontinuation of dabigatran. The pooled data in this study from a large sample of patients provides an opportunity to address some of these issues.

In this meta-analysis comprising 14 controlled trials, dabigatran performed similarly to warfarin with regard to major bleeding and thromboembolism. Moreover, no deaths were observed in any of the treatment strategies. These results are in agreement with the favourable profile that was observed in an early observational non-controlled trial by Winckle et al. ${ }^{30}$ Furthermore, a recent analysis of the US Food and Drug Administration (FDA) Mini-Sentinel database for the period from 19 October 2010 (the date of dabigatran approval) to 31 December 2011, found that bleeding rates associated with dabigatran use during the period of interest did not appear to be higher than those associated with warfarin. ${ }^{31}$

Though the present analysis suggests a lower rate of minor bleeding with dabigatran, there were pronounced differences in the reporting of this end point across the different studies. Furthermore, on sensitivity analysis these results did not remain significant. Thus, while this result needs to be interpreted with caution, it seems, that at the very least, dabigatran is unlikely to be inferior to warfarin in this regard.

No significant differences in major bleeding and thromboembolism were observed between the two assigned dabigatran dosages. However, the low number of patients in each group (resulting from under-reporting of the dosage used in most selected studies), and the low rate of observed events, limit the extent to which firm conclusions can be drawn. The suggestion of less frequent minor bleeding with the $150 \mathrm{mg}$ twice daily dosage is hard to interpret due to the differences in reporting of this end point and in sample size.

The various transition regimens used in patients treated with dabigatran, and the low number of observed events in each of those, renders difficult the task of establishing an ideal transition regimen in this setting. However, the great inter- and sometimes intra-study variability notwithstanding, certain factors are likely to be important for decision making. 
Table 4 Comparison of endpoints across studies

\begin{tabular}{|c|c|c|c|c|c|c|c|c|c|c|c|c|c|c|}
\hline & $\begin{array}{l}\text { Bassiouny } \\
\text { et } a l^{15}\end{array}$ & $\begin{array}{l}\text { Bernard } \\
\text { et } \mathrm{al}^{23}\end{array}$ & $\begin{array}{l}\text { Haines } \\
\text { et } a l^{16}\end{array}$ & $\begin{array}{l}\text { Ichiki } \\
\text { et } a^{24}\end{array}$ & $\begin{array}{l}\text { Kaseno } \\
\text { et } a l^{17}\end{array}$ & Kim et $a l^{18}$ & $\begin{array}{l}\text { Lakkireddy } \\
\text { et } a l^{9}\end{array}$ & $\begin{array}{l}\text { Maddox } \\
\text { et } a l^{19}\end{array}$ & $\begin{array}{l}\text { Mendoza } \\
\text { et } \mathrm{al}^{25}\end{array}$ & Nin et al ${ }^{20}$ & $\begin{array}{l}\text { Pavaci } \\
\text { et } a l^{26}\end{array}$ & $\begin{array}{l}\text { Rowley } \\
\text { et al. }{ }^{27}\end{array}$ & $\begin{array}{l}\text { Snipelisky } \\
\text { et } a^{21}\end{array}$ & $\begin{array}{l}\text { Yamaji } \\
\text { et }\left.a\right|^{22}\end{array}$ \\
\hline Minor bleeding & $\begin{array}{l}7 / 376 \mathrm{D} \\
16 / 623 \mathrm{~W} \\
\text { Groin } \\
\text { haematoma } \\
5 / 376 \mathrm{D} \\
10 / 623 \mathrm{~W} \\
\text { Gl bleed } \\
1 / 376 \mathrm{D} \\
2 / 623 \mathrm{~W} \\
\text { Minor } \\
\text { haemoptysis } \\
1 / 623 \mathrm{~W} \\
\text { Epistaxis1/ } \\
623 \mathrm{~W} \\
\text { PEff without } \\
\text { tamponade } \\
1 / 376 \mathrm{D} \\
2 / 623 \mathrm{~W}\end{array}$ & $\begin{array}{l}1 / 155 \mathrm{D} \\
2 / 44 \mathrm{~W}\end{array}$ & $\begin{array}{l}\text { 4/ } 222 \mathrm{D} \\
\text { 2/ } 222 \mathrm{~W} \\
\text { Minor } \\
\text { bleeding } \\
\text { complications } \\
\text { 2/ 222 D } \\
1 / 222 \mathrm{~W} \\
\text { Vascular } \\
\text { complication } \\
\text { 2/222 D } \\
\text { 1/222 W }\end{array}$ & NA & $\begin{array}{l}5 / 110 \mathrm{D} \\
11 / 101 \mathrm{~W} \\
\text { Groin } \\
\text { haematoma } \\
5 / 110 \mathrm{D} \\
11 / 101 \mathrm{~W}\end{array}$ & $\begin{array}{l}\text { 5/191 D } \\
19 / 572 \mathrm{~W} \\
\text { Groin } \\
\text { haematoma } \\
\text { 4/191 D } \\
\text { 19/572 W } \\
\text { PEff without } \\
\text { tamponade } \\
\text { 1/191 D }\end{array}$ & $\begin{array}{l}12 / 145 \mathrm{D} \\
8 / 145 \mathrm{~W} \\
\text { Groin } \\
\text { haematoma } \\
6 / 145 \mathrm{D} \\
5 / 145 \mathrm{~W} \\
\text { PEff without } \\
\text { tamponade } \\
6 / 145 \mathrm{D} \\
4 / 145 \mathrm{~W}\end{array}$ & $\begin{array}{l}1 / 212 \mathrm{D} \\
3 / 251 \mathrm{~W} \\
\text { Groin } \\
\text { haematoma } \\
1 / 212 \mathrm{D} \\
\text { 2/251 W } \\
\text { Minor } \\
\text { haemoptysis } \\
\text { 1/251 W }\end{array}$ & $\begin{array}{l}1 / 60 \mathrm{D} \\
1 / 58 \mathrm{~W} \\
\text { Groin } \\
\text { haematoma } \\
1 / 58 \mathrm{~W} \\
\text { GI bleed } \\
1 / 60 \mathrm{D}\end{array}$ & $\begin{array}{l}\text { 9/45 D } \\
\text { 20/45 W } \\
\text { Rebleeding from } \\
\text { puncture site } \\
\text { 9/45 D } \\
20 / 45 \mathrm{~W}\end{array}$ & $\begin{array}{l}2 / 27 \mathrm{D} \\
4 / 27 \mathrm{~W} \\
\text { Groin } \\
\text { haematoma } \\
2 / 27 \mathrm{D} \\
4 / 27 \mathrm{~W}\end{array}$ & $\begin{array}{l}5 / 113 \mathrm{D} \\
33 / 169 \mathrm{~W} \\
\text { Non-life } \\
\text { threatening } \\
\text { vascular } \\
\text { complications } \\
5 / 113 \mathrm{D} \\
\text { 33/169 W }\end{array}$ & $\begin{array}{l}6 / 31 \mathrm{D} \\
21 / 125 \mathrm{~W} \\
\text { Haematoma } \\
1 / 31 \mathrm{D} \\
4 / 125 \mathrm{~W} \\
\text { Rebleeding } \\
5 / 31 \mathrm{D} \\
10 / 125 \mathrm{~W} \\
\text { Ecchymosis } \\
7 / 125 \mathrm{~W}\end{array}$ & $\begin{array}{l}2 / 106 \mathrm{D} \\
11 / 397 \mathrm{~V} \\
\text { Groin } \\
\text { haemator } \\
1 / 106 \mathrm{D} \\
4 / 397 \mathrm{~W} \\
\text { PEff } \\
\text { without } \\
\text { tampona } \\
1 / 106 \mathrm{D} \\
7 / 397 \mathrm{~W}\end{array}$ \\
\hline Major bleeding & $\begin{array}{l}\text { 4/376 D } \\
8 / 623 \mathrm{~W} \\
\text { PEff with } \\
\text { need of pct } \\
\text { 3/ D } \\
\text { 7/ W } \\
\text { Haemoptysis* } \\
\text { 1/ W } \\
\text { Cerebral } \\
\text { bleeding } \\
\text { 1/ Dt }\end{array}$ & $\begin{array}{l}2 / 155 \mathrm{D} \\
2 / 44 \mathrm{~W}\end{array}$ & $\begin{array}{l}3 / 222 \mathrm{D} \\
3 / 222 \mathrm{~W} \\
\text { PEff with } \\
\text { need of pct } \\
2 / 222 \mathrm{D} \\
2 / 222 \mathrm{~W} \\
\text { Other major } \\
\text { bleeding } \\
1 / 222 \mathrm{D} \\
1 / 222 \mathrm{~W}\end{array}$ & $\begin{array}{l}4 / 30 \mathrm{D} \\
4 / 180 \\
\text { W } \\
\text { PEff } \\
\text { with } \\
\text { need } \\
\text { of pct } \\
4 / 30 \mathrm{D} \\
4 / 180 \\
\text { W }\end{array}$ & $\begin{array}{l}0 / 110 \mathrm{D} \\
2 / 101 \mathrm{~W} \\
\text { Cardiac } \\
\text { tamponade } \\
2 / 101 \mathrm{~W}\end{array}$ & $\begin{array}{l}4 / 191 \mathrm{D} \\
12 / 572 \mathrm{~W} \\
\text { Cardiac } \\
\text { tamponade } \\
2 / 191 \mathrm{D} \\
7 / 572 \mathrm{~W} \\
\text { Vascular }\end{array}$ & & $\begin{array}{l}\text { complications } \\
\text { 2/191 D } \\
5 / 572 \mathrm{~W}\end{array}$ & $\begin{array}{l}9 / 145 \mathrm{D} \\
1 / 145 \mathrm{~W} \\
\text { Cardiac } \\
\text { tamponade } \\
9 / 145 \mathrm{D} \\
1 / 145 \mathrm{~W}\end{array}$ & $\begin{array}{l}1 / 212 \mathrm{D} \\
3 / 251 \mathrm{~W} \\
\text { Cardiac tamponade } \\
1 / 212 \mathrm{D} \\
2 / 251 \mathrm{~W} \\
\text { Retroperitoneal } \\
\text { haematoma } \\
1 / 251 \mathrm{~W}\end{array}$ & 0 & 0 & 0 & $\begin{array}{l}0 / 113 \mathrm{D} \\
1 / 169 \mathrm{~W}\end{array}$ \\
\hline $\begin{array}{l}\text { Retroperitoneal } \\
\text { bleed } \\
1 / 169 \mathrm{~W}\end{array}$ & 0 & $\begin{array}{l}\text { 0/106 D } \\
4 / 397 \mathrm{~W} \\
\text { Cardiac }\end{array}$ & $\begin{array}{l}\text { tamponade } \\
4 / 397 \mathrm{~W}\end{array}$ & & & & & & & & & & & \\
\hline Thrombo-embolism & $\begin{array}{l}1 / 376 \mathrm{D} \\
1 / 623 \mathrm{~W} \\
\text { Stroke } \\
1 / 623 \mathrm{~W} \\
\text { Pulmonary } \\
\text { embolism } \\
1 / 376 \mathrm{D}\end{array}$ & 0 & $\begin{array}{l}2 / 222 \mathrm{D} \neq \\
0 / 222 \mathrm{~W} \\
\text { Stroke } \\
\text { 1/222 D } \\
\text { TIA } \\
\text { 1/222 D }\end{array}$ & 0 & 0 & 0 & $\begin{array}{l}3 / 145 \mathrm{D} \\
0 / 145 \mathrm{~W} \\
\text { Stroke or } \\
\text { TIA } \\
3 / 145 \mathrm{D}\end{array}$ & $\begin{array}{l}1 / 212 \mathrm{D} \\
0 / 251 \\
\text { TIA } \\
1 / 212 \mathrm{D}\end{array}$ & $\begin{array}{l}0 / 60 \mathrm{D} \\
1 / 58 \mathrm{~W} \\
\text { Stroke } \\
1 / 58 \mathrm{~W}\end{array}$ & $\begin{array}{l}0 / 45 \mathrm{D} \\
1 / 45 \mathrm{~W} \\
\text { Mesenteric } \\
\text { thrombosis } \\
1 / 45 \mathrm{~W}\end{array}$ & $\begin{array}{l}1 / 27 \mathrm{D} \\
0 / 27 \mathrm{~W} \\
\text { Stroke } \\
1 / 27 \mathrm{D}\end{array}$ & $\begin{array}{l}2 / 113 \mathrm{D} \\
2 / 169 \mathrm{~W} \\
\text { Stroke } \\
1 / 113 \mathrm{D} \\
1 / 169 \mathrm{~W} \\
\text { TIA } \\
\text { 1/113 D } \\
\text { Pulmonary } \\
\text { embolism } \\
\text { 1/169 W }\end{array}$ & 0 & 0 \\
\hline
\end{tabular}

*Requiring transfusion.

Patient with a cerebral cavernous malformation

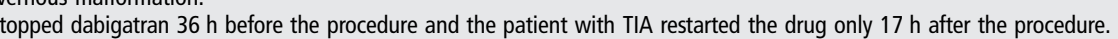

D, dabigatran; GI, gastrointestinal; NA, data not available; pct, pericardiocentesis; PEff, pericardial effusion; punct, puncture; TIA, transient ischaemic attack; W, warfarin. 
Figure 3 Funnel plots representing the studies used in the assessment of: (A) stroke and thromboembolism; and (B) major bleeding. The inverted and symmetrical funnel aspect can be observed for the assessed end points, with $95 \%$ of the studies lying within the confidence limit lines. This suggests that publication bias is not present among the selected studies for the meta-analysis.
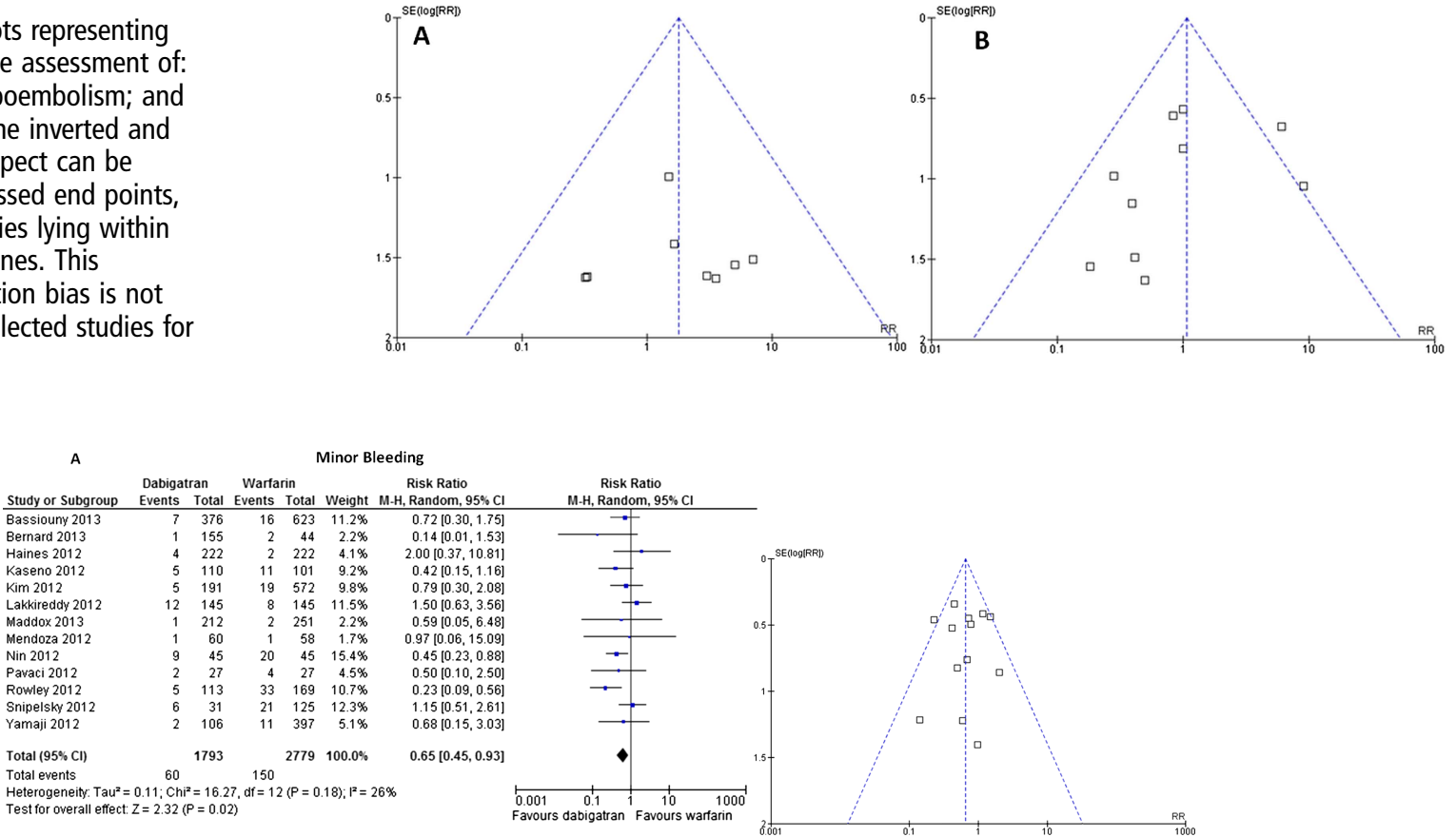

Figure 4 Incidence of minor bleeding among patients treated with dabigatran or warfarin. (A) Forest plot. (B) Funnel-plot. M-H, Mantel-Haenszel.

A

Stroke and Thromboembolism

Risk Ratio

B

Stroke and Thromboembolism

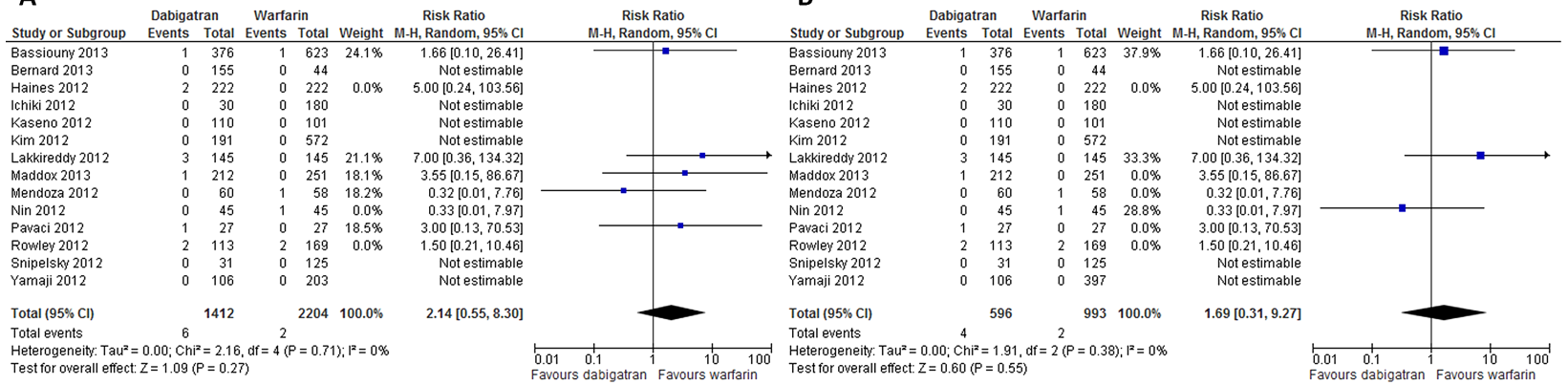

Major Bleeding

\begin{tabular}{|c|c|c|c|c|c|c|c|c|}
\hline \multirow[b]{2}{*}{ Study or Subgroup } & & \multirow{2}{*}{\multicolumn{2}{|c|}{$\begin{array}{c}\text { Risk Ratio } \\
\text { M-H, Random, } 95 \% \mathrm{Cl}\end{array}$}} \\
\hline & \multicolumn{2}{|c|}{ Dabigatran } & \multicolumn{2}{|c|}{ Warfarin } & \multicolumn{2}{|c|}{$\begin{array}{c}\text { Risk Ratio } \\
\text { Weight } \\
\text { M.H, Random, } 95 \% \mathrm{Cl}\end{array}$} & & \\
\hline Bassiouny 2013 & 4 & 376 & 8 & 623 & $17.9 \%$ & $0.83[0.25,2.73]$ & & \\
\hline Bernard 2013 & 2 & 155 & 2 & 44 & $12.0 \%$ & $0.28[0.04,1.96]$ & & - \\
\hline Haines 2012 & 3 & 222 & 3 & 222 & $0.0 \%$ & $1.00[0.20,4.90]$ & & \\
\hline Ichiki 2012 & 4 & 30 & 4 & 180 & $16.7 \%$ & $6.00[1.59,22.71]$ & & \\
\hline Kaseno 2012 & 0 & 110 & 2 & 101 & $6.8 \%$ & $0.18[0.01,3.78]$ & & \\
\hline Kim 2012 & 4 & 191 & 12 & 572 & $18.6 \%$ & $1.00[0.33,3.06]$ & & - \\
\hline Lakkiredd & 9 & 145 & 1 & 145 & $11.2 \%$ & $9.00[1$ & & \\
\hline Maddo 2 & 1 & 212 & 3 & 251 & $10.0 \%$ & $0.39[0.04,3$ & & \\
\hline Mend & 0 & 60 & 0 & 58 & & Not estima & & \\
\hline $\operatorname{Nin} 2$ & 0 & 45 & 0 & 45 & & Not estil & & \\
\hline Pavaci 2 & 0 & 27 & 0 & 27 & & Not estir & & \\
\hline D & 0 & 113 & 1 & 169 & $0.0 \%$ & $0.50[0.02$ & & \\
\hline & 0 & 31 & 0 & 125 & & Note & & \\
\hline Yamaji 2012 & 0 & 106 & 2 & 203 & $6.7 \%$ & $0.38[0.02,7.87]$ & & \\
\hline otal (95\% & & 1412 & & 2204 & $100.0 \%$ & $1.09[0.44,2.72]$ & & \\
\hline Total events & & & 34 & & & & & \\
\hline $\begin{array}{l}\text { Heterogeneity: } \mathrm{Tau}^{2}= \\
\text { Test for overall effect. }\end{array}$ & $\begin{array}{l}0.84 ; \mathrm{Ch}^{2} \\
\mathrm{z}=0.19\end{array}$ & $\begin{array}{l}2=14.8 \\
P=0.85\end{array}$ & & & & & $\begin{array}{l}0.01 \\
\text { Favours }\end{array}$ & 1 \\
\hline
\end{tabular}

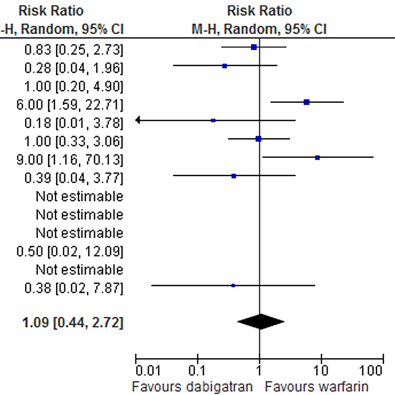

Major Bleeding
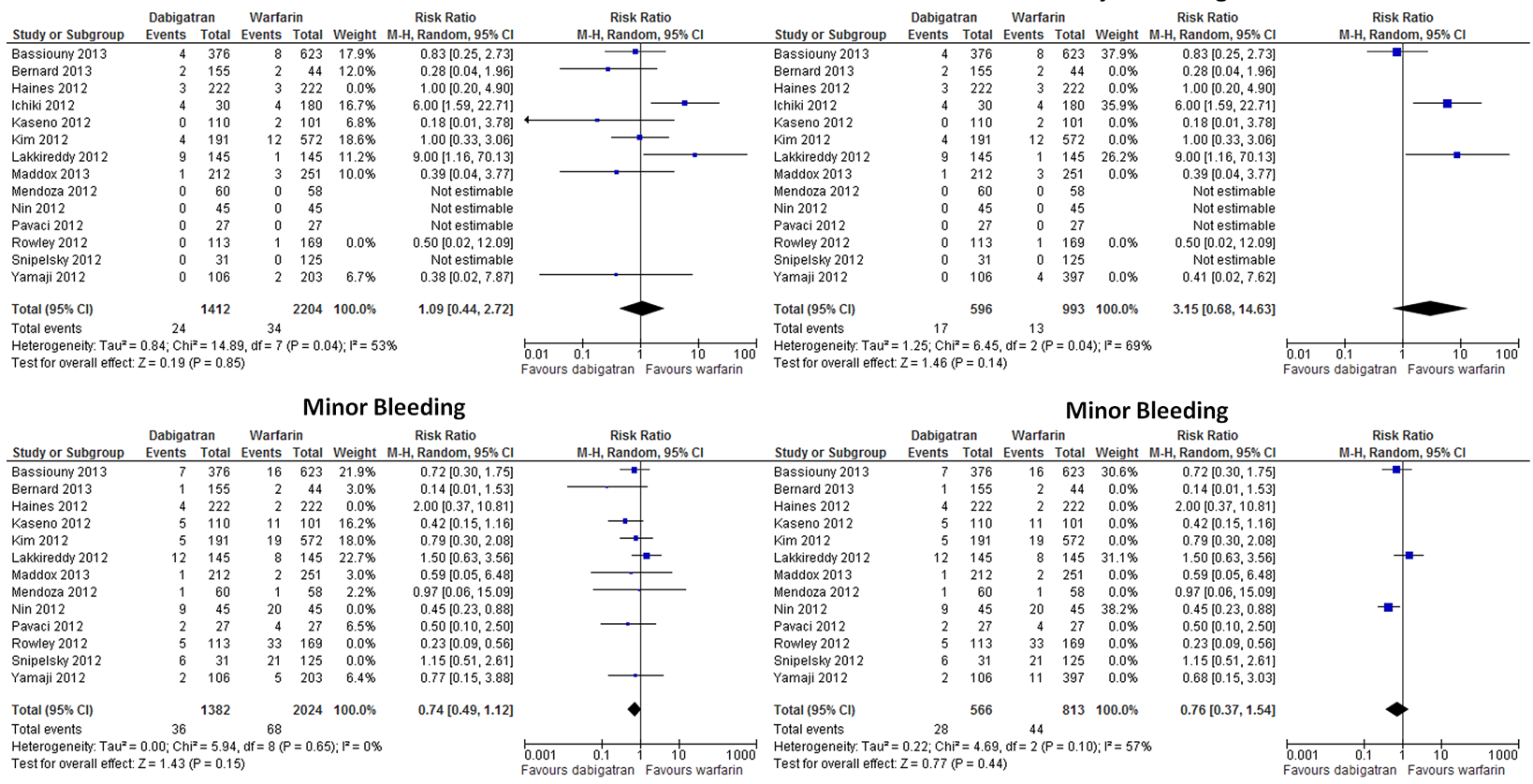

Minor Bleeding

Figure 5 Forest plot illustrating the sensitivity analysis restricting data to: (A) trials whose patients were treated uninterrupted warfarin; and (B) prospective studies. M-H, Mantel-Haenszel. 


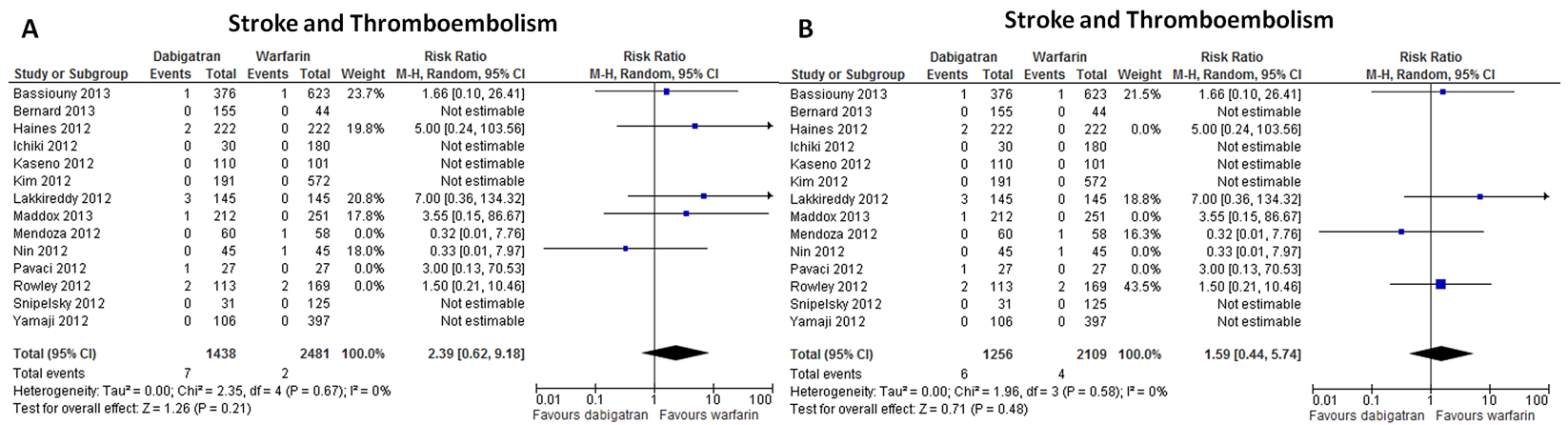

Figure 6 Forest plot illustrating the sensitivity analysis restricting data to: (A) investigations published as full text articles; and (B) studies whose follow-up was at least 30 days. M-H, Mantel-Haenszel.

The timing for drug interruption before the procedure must take into account the patient's renal function, as $80 \%$ of the drug is excreted by this pathway.

In patients with normal renal clearance, the best option may be drug suspension on the morning of the procedure, or the night before, but always $<24 \mathrm{~h}$ before the procedure.

If renal function is compromised, the drug should probably be interrupted sooner, depending on the degree of renal dysfunction.

There may be a rationale for restarting dabigatran $3-4 \mathrm{~h}$ after assuring haemostasis, considering its short half-life and rapid onset of action.

The target ACT during catheter ablation in patients treated with dabigatran should be between $300-350 \mathrm{~s}$ (similar to warfarin), as no additional benefits were found for higher values.

Finally, as there seems to be no inherent advantage of any of the studied dabigatran dosages, the creatinine clearance and bleeding risk (HAS-BLED score) should be taken into account for deciding the dosage to be used.

\section{LIMITATIONS}

Important strengths of this study include the consistent reporting for thromboembolism (almost absent heterogeneity, $\mathrm{I}^{2}=0 \%$ ) across studies. However, there are certain limitations, inherent to any meta-analysis.
First, heterogeneity was observed regarding major bleeding $\left(\mathrm{I}^{2}=41 \%\right.$; moderate heterogeneity). This may be explained by the pronounced diversity in ablation strategy, anticoagulation regimen, intraprocedural use of heparin, and follow-up between the incorporated studies. However, heterogeneity was low $\left(\mathrm{I}^{2}=12 \%\right)$ in the sensitivity analysis including only higher methodological quality studies, which confirms the small and similar incidence of major bleeding in both treatment arms already suggested by the forest plot that included all studies.

Secondly, as already highlighted, certain comparisons are limited by the low number of subjects and low event rates.

Thirdly, in some cases of abstract related data, even with the best attempts to gather information by directly contacting the authors, some data remained incomplete. However, these were mainly with regard to minor bleeding (a secondary end point) and details concerning periprocedural drug transition; hence, missing data are unlikely to have significantly affected the primary results of the analysis.

Fourthly, only a minority of trials was randomised or prospective and a blinded analysis and/or central adjudication of end points was performed in none (ie, the higher level of data quality). Therefore, these data must be considered preliminary and interpreted with some caution. 
Stroke and Thromboembolism

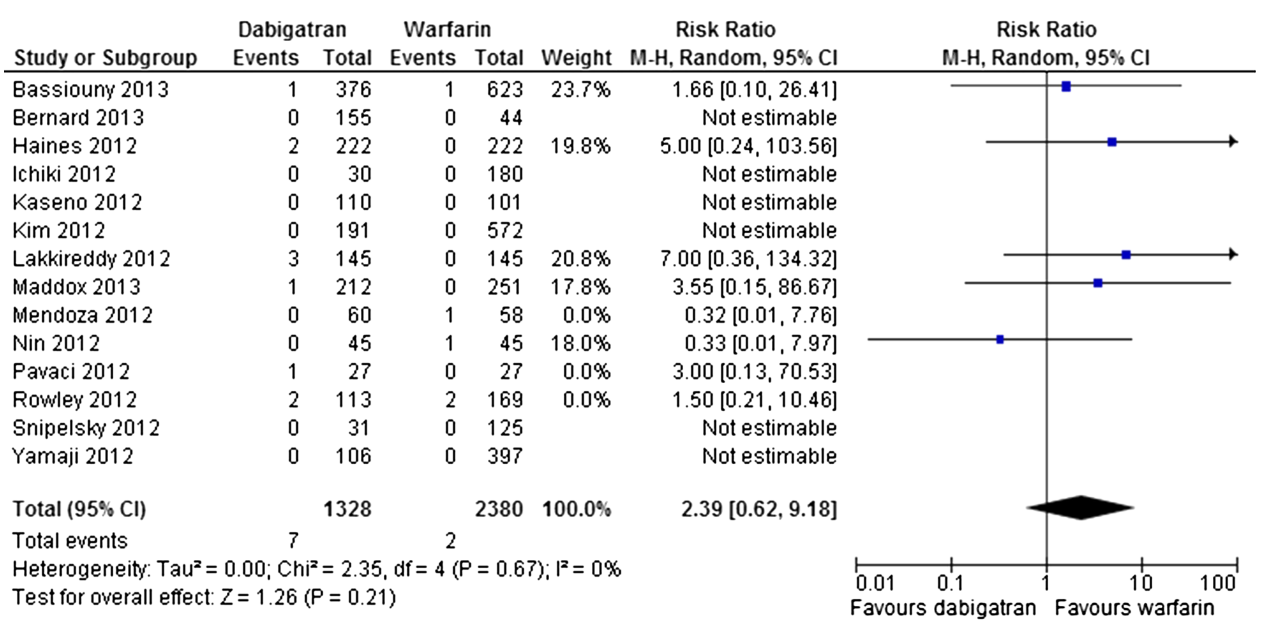

\section{Major Bleeding}

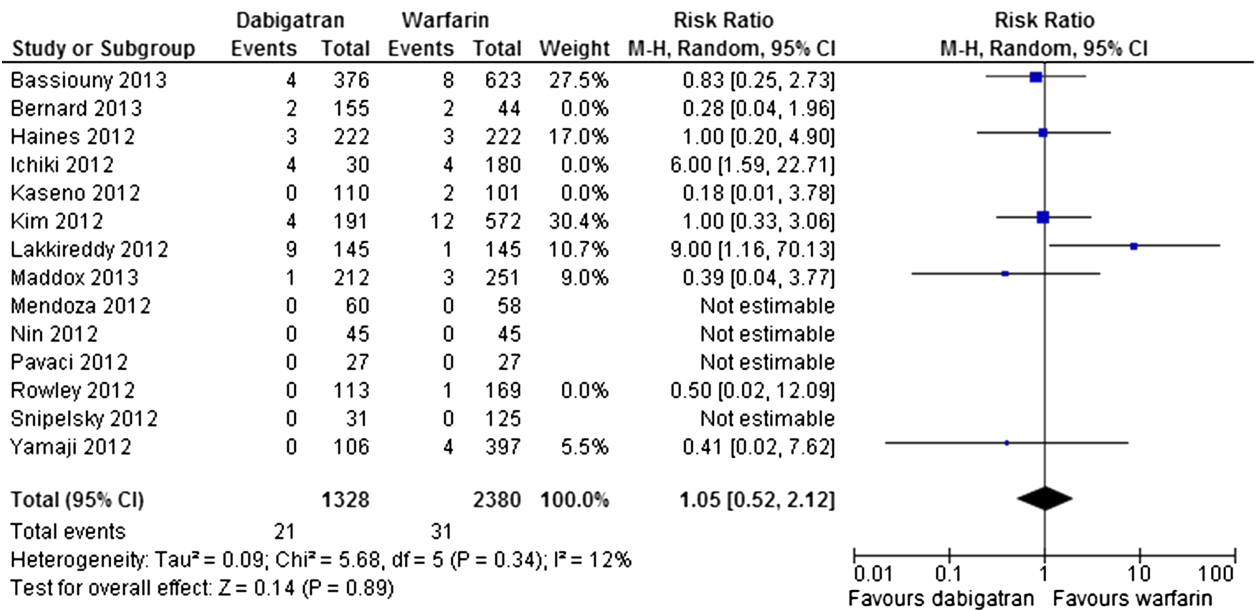

\section{Minor Bleeding}

\begin{tabular}{|c|c|c|c|c|c|c|c|c|}
\hline \multirow[b]{2}{*}{ Study or Subgroup } & \multicolumn{2}{|c|}{ Dabigatran } & \multicolumn{2}{|c|}{ Warfarin } & \multirow[b]{2}{*}{ Weight } & \multirow{2}{*}{$\begin{array}{c}\text { Risk Ratio } \\
\mathrm{M}-\mathrm{H}, \text { Random, } 95 \% \mathrm{Cl}\end{array}$} & \multirow{2}{*}{$\begin{array}{c}\text { Risk Ratio } \\
\mathrm{M}-\mathrm{H}, \text { Random, } 95 \% \mathrm{Cl}\end{array}$} & \\
\hline & Events & Total & Events & Total & & & & \\
\hline Bassiouny 2013 & 7 & 376 & 16 & 623 & $15.4 \%$ & $0.72[0.30,1.75]$ & $\rightarrow$ & \\
\hline Bernard 2013 & 1 & 155 & 2 & 44 & $0.0 \%$ & $0.14[0.01,1.53]$ & & \\
\hline Haines 2012 & 4 & 222 & 2 & 222 & $4.2 \%$ & $2.00[0.37,10.81]$ & & \\
\hline Kaseno 2012 & 5 & 110 & 11 & 101 & $0.0 \%$ & $0.42[0.15,1.16]$ & & \\
\hline Kim 2012 & 5 & 191 & 19 & 572 & $12.6 \%$ & $0.79[0.30,2.08]$ & & \\
\hline Lakkireddy 2012 & 12 & 145 & 8 & 145 & $15.9 \%$ & $1.50[0.63,3.56]$ & $\leftarrow$ & \\
\hline Maddox 2013 & 1 & 212 & 2 & 251 & $2.1 \%$ & $0.59[0.05,6.48]$ & & \\
\hline Mendoza 2012 & 1 & 60 & 1 & 58 & $0.0 \%$ & $0.97[0.06,15.09]$ & & \\
\hline $\operatorname{Nin} 2012$ & 9 & 45 & 20 & 45 & $26.6 \%$ & $0.45[0.23,0.88]$ & $\rightarrow-$ & \\
\hline Pavaci 2012 & 2 & 27 & 4 & 27 & $0.0 \%$ & $0.50[0.10,2.50]$ & & \\
\hline Rowley 2012 & 5 & 113 & 33 & 169 & $0.0 \%$ & $0.23[0.09,0.56]$ & & \\
\hline Snipelsky 2012 & 6 & 31 & 21 & 125 & $17.8 \%$ & $1.15[0.51,2.61]$ & & \\
\hline Yamaji 2012 & 2 & 106 & 11 & 397 & $5.4 \%$ & $0.68[0.15,3.03]$ & & \\
\hline Total $(95 \% \mathrm{Cl})$ & & 1328 & & 2380 & $100.0 \%$ & $0.82[0.58,1.15]$ & & \\
\hline Total events & 46 & & 99 & & & & & \\
\hline \multicolumn{7}{|c|}{$\begin{array}{l}\text { Heterogeneity: } \mathrm{Tau}^{2}=0.00 ; \mathrm{Chi}^{2}=6.92, \mathrm{df}=7(P=0.44) ; \mathrm{I}^{2}=0 \% \\
\text { Test for overall effect: } Z=1.16(P=0.25)\end{array}$} & $\begin{array}{ll}1 & 10 \\
1 & 10\end{array}$ & 1000 \\
\hline
\end{tabular}

Figure 7 Forest plot illustrating the sensitivity analysis restricting data to higher quality full text articles (Delphi criteria or Newcastle-0ttawa Scale $\geq 5$ ). M-H, Mantel-Haenszel.

\section{CONCLUSIONS}

The rate of thromboembolic complications and/or major bleeding in patients on dabigatran undergoing AF catheter ablation is low and similar to that seen with warfarin.

These results may suggest the feasibility and safety of AF catheter ablation in patients regularly treated with dabigatran. However, further prospective and randomised studies are still necessary to confirm these findings and clarify which periprocedural regimen can minimise the risk of thromboembolic complications with dabigatran.

Contributors RP, SB were involved in the conception and design of the study. RP, $A B$ and $B C$ collected all data. RP, JPA, SC, AB, BC, NC, KN, EM and SB have critically revised the obtained data and provided suggestions for the preparation of the first version of the manuscript. RP, SB, EM, KN wrote the first version of the 
manuscript. RP, JPA, SC, AB, BC, NC, KN, EM and SB carefully and critically revised this version and suggested the necessary changes for its improvement. The final version of the manuscript was revised by RP, JPA, SC, AB, BC, NC, KN, EM and SB and a group discussion was conducted with all authors before the final approval of the manuscript.

Competing interests RP has received honoraria for serving as a speaker and consultant for Boehringer-Ingelheim and as a co-investigator in the ENGAGE-AF TIMI 48 trial. There are no conflicts of interest for other co-authors.

Provenance and peer review Not commissioned; externally peer reviewed.

Open Access This is an Open Access article distributed in accordance with the Creative Commons Attribution Non Commercial (CC BY-NC 3.0) license, which permits others to distribute, remix, adapt, build upon this work non-commercially, and license their derivative works on different terms, provided the original work is properly cited and the use is non-commercial. See: http://creativecommons.org/ licenses/by-nc/3.0/

\section{REFERENCES}

1 Chen LY, Shen WK. Epidemiology of atrial fibrillation: a current perspective. Heart Rhythm 2007:4(3 Suppl):S1-6.

2 Lin $\mathrm{HJ}$, Wolf PA, Kelly-Hayes M, et al. Stroke severity in atrial fibrillation. The Framingham Study. Stroke 1996;27:1760-4.

3 Wann LS, Curtis AB, January CT, et al. 2011 ACCF/AHA/HRS focused update on the management of patients with atrial fibrillation (updating the 2006 Guideline): a report of the American College of Cardiology Foundation/American Heart Association Task Force on Practice Guidelines. Heart Rhythm 2011;8:157-76.

4 Crawford T, Oral H. Current status and outcomes of catheter ablation for atrial fibrillation. Heart Rhythm 2009;6(12 Suppl):S12-17.

5 Connolly SJ, Ezekowitz MD, Yusuf S, et al. Dabigatran versus warfarin in patients with atrial fibrillation. N Engl J Med 2009;361:1139-51.

6 Patel MR, Mahaffey KW, Garg J, et al. Rivaroxaban versus warfarin in nonvalvular atrial fibrillation. N Engl J Med 2011;365:883-91.

7 Granger $\mathrm{CB}$, Alexander JH, McMurray JJ, et al. Apixaban versus warfarin in patients with atrial fibrillation. N Engl J Med 2011;365:981-92.

8 Calkins H, Kuck KH, Cappato R, et al. 2012 HRS/EHRA/ECAS expert consensus statement on catheter and surgical ablation of atrial fibrillation: recommendations for patient selection, procedural techniques, patient management and follow-up, definitions, endpoints, and research trial design. Heart Rhythm 2012;9:632-96.e21.

9 Lakkireddy D, Reddy YM, Di Biase L, et al. Feasibility and safety of dabigatran versus warfarin for periprocedural anticoagulation in patients undergoing radiofrequency ablation for atrial fibrillation: results from a multicenter prospective registry. J Am Coll Cardiol 2012;59:1168-74.

10 Menzies D. Systematic reviews and meta-analyses. Int I Tuberc Lung Dis 2011;15:582-93

11 Moher D, Liberati A, Tetzlaff J, et al. PRISMA Group: preferred reporting items for systematic reviews and meta-analyses: the PRISMA statement. Ann Intern Med 2009;151:264-9.

12 Verhagen AP, de Vet HC, de Bie RA, et al. The Delphi list: a criteria list for quality assessment of randomized clinical trials for conducting systematic reviews developed by Delphi consensus. J Clin Epidemiol 1998;51:1235-41.

13 Wells G, Shea B, O'Connell D, et al. The Newcastle-Ottawa Scale (NOS) for assessing the quality of nonrandomised studies in meta-analyses. http://www.ohri. ca/programs/clinical_epidemiology/oxford.htm (accessed 28 Jun 2013).
14 Sorgente A, Chierchia GB, de Asmundis C, et al. Complications of atrial fibrillation ablation: when prevention is better than cure. Europace 2011;13:1526-32.

15 Bassiouny M, Saliba W, Rickard J, et al. Use of dabigatran for peri-procedural anticoagulation in patients undergoing catheter ablation for atrial fibrillation. Circ Arrhythm Electrophysiol. 2013;6:460-6.

16 Haines DE, Mead-Salley M, Salazar M, et al. Dabigatran versus warfarin anticoagulation before and after catheter ablation for the treatment of atrial fibrillation. I Interv Card Electrophysiol. 2013. [Epub ahead of print]

17 Kaseno K, Naito S, Nakamura K, et al. Efficacy and safety of periprocedural dabigatran in patients undergoing catheter ablation of atrial fibrillation. Circ $J$ 2012;76:2337-42.

$18 \mathrm{Kim}$ JS, She F, Jongnarangsin K, et al. Dabigatran vs warfarin for radiofrequency catheter ablation of atrial fibrillation. Heart Rhythm 2013:10:483-9.

19 Maddox W, Kay GN, Yamada T, et al. Dabigatran versus warfarin therapy for uninterrupted oral anticoagulation during atrial fibrillation ablation. J Cardiovasc Electrophysiol 2013. doi: 10.1111/jce.12143. [Epub ahead of print]

20 Nin T, Sairaku A, Yoshida Y, et al. A Randomized controlled trial of dabigatran versus warfarin for periablation anticoagulation in patients undergoing ablation of atrial fibrillation. Pacing Clin Electrophysiol 2013;36:172-9.

21 Snipelisky D, Kauffman C, Prussak K, et al. A comparison of bleeding complications post-ablation between warfarin and dabigatran. J Interv Card Electrophysiol 2012:35:29-33.

22 Yamaji H, Murakami T, Hina K, et al. Usefulness of dabigatran etexilate as periprocedural anticoagulation therapy for atrial fibrillation ablation. Clin Drug Investig 2013;33:409-18.

23 Bernard $M$, Brabham W, Netzler $\mathrm{P}$, et al. Comparison of atrial fibrillation ablation bleeding and thrombotic complications with dabigatran, rivaroxaban and warfarin J Am Coll Cardiol 2013;61:(Suppl):E276. Abstract.

24 Ichiki H, Oketani N, Iriki Y, et al. The comparison of warfarin and dabigatran in the incidence of asymptomatic cerebral micro thromboembolism after AF ablation. Circulation 2012;126(21 Suppl 1):A9301. Abstract.

25 Mendoza I, Helguera M, Baez-Escudero J, et al. Atrial fibrillation ablation on uninterrupted anticoagulation with dabigatran versus warfarin. Heart Rhythm 2012;9(5 Suppl 1):S270-1. Abstract.

26 Pavaci H, Reents T, Ammar S, et al. Safety and efficacy of dabigatran in patients undergoing left atrial ablation procedures: a case-matched analysis. Eur Heart J 2012;33(Abstract Supplement):60-1. Abstract.

27 Rowley CP, Bradford NS, Bernard ML, et al. Complications of atrial fibrillation ablation in patients anticoagulated with dabigatran compared to warfarin. Heart Rhythm 2012;9(5 Suppl 1):S201. Abstract.

28 Piccini JP, Sinner MF, Greiner MA, et al. Outcomes of Medicare beneficiaries undergoing catheter ablation for atrial fibrillation. Circulation 2012; 126:2200-7.

29 Hussein AA, Martin DO, Saliba W, et al. Radiofrequency ablation of atrial fibrillation under therapeutic international normalized ratio: a safe and efficacious periprocedural anticoagulation strategy. Heart Rhythm 2009;6:1425-9.

30 Winkle RA, Mead RH, Engel $\mathrm{G}$, et al. The use of dabigatran immediately after atrial fibrillation ablation. J Cardiovasc Electrophysiol 2012;23:264-8.

31 Ross Southworth M, Reichman ME, Unger EF. Dabigatran and postmarketing reports of bleeding. N Engl J Med 2013;368:1272-4. 